\title{
THE SPITZER MID-INFRARED ACTIVE GALACTIC NUCLEUS SURVEY. I. OPTICAL AND NEAR-INFRARED SPECTROSCOPY OF OBSCURED CANDIDATES AND NORMAL ACTIVE GALACTIC NUCLEI SELECTED IN THE MID-INFRARED
}

\author{
M. Lacy ${ }^{1}$, S. E. Ridgway ${ }^{2}$, E. L. Gates ${ }^{3}$, D. M. Nielsen ${ }^{4}$, A. O. Petric ${ }^{5}$, A. Sajina ${ }^{6}$, T. Urrutia ${ }^{7}$, \\ S. Cox Drews ${ }^{8}$, C. Harrison ${ }^{9}$, N. Seymour ${ }^{10}$, AND L. J. Storrie-Lombardi ${ }^{11}$ \\ ${ }^{1}$ National Radio Astronomy Observatory, 520 Edgemont Road, Charlottesville, VA 22903, USA \\ ${ }^{2}$ National Optical Astronomy Observatory, 950 North Cherry Avenue, Tucson, AZ 85719, USA \\ ${ }^{3}$ UCO/Lick Observatory, P.O. Box 85, Mount Hamilton, CA 95140, USA \\ ${ }^{4}$ Department of Astronomy, University of Wisconsin, 475 N. Charter Street, Madison, WI 53706, USA \\ ${ }^{5}$ Department of Astronomy, California Institute of Technology, Pasadena, CA 91125, USA \\ ${ }^{6}$ Department of Physics and Astronomy, Tuffs University, 212 College Avenue, Medford, MA 02155, USA \\ ${ }^{7}$ Leibniz-Institut für Astrophysik Potsdam, An der Sternwarte 16, D-14482 Potsdam, Germany \\ 8946 Mangrove Avenue \#102, Sunnyvale, CA 94086, USA \\ ${ }^{9}$ Department of Astronomy, University of Michigan, Ann Arbor, MI 48109, USA \\ ${ }^{10}$ CSIRO, P.O. Box 76, Epping, NSW 1710, Australia \\ 11 Spitzer Science Center, California Institute of Technology, Pasadena, CA 91125, USA \\ Received 2013 April 23; accepted 2013 August 15; published 2013 September 27
}

\begin{abstract}
We present the results of a program of optical and near-infrared spectroscopic follow-up of candidate active galactic nuclei (AGNs) selected in the mid-infrared. This survey selects both normal and obscured AGNs closely matched in luminosity across a wide range, from Seyfert galaxies with bolometric luminosities $L_{\text {bol }} \sim 10^{10} L_{\odot}$ to highly luminous quasars $\left(L_{\text {bol }} \sim 10^{14} L_{\odot}\right)$, all with redshifts ranging from 0 to 4.3. Samples of candidate AGNs were selected with mid-infrared color cuts at several different $24 \mu \mathrm{m}$ flux density limits to ensure a range of luminosities at a given redshift. The survey consists of 786 candidate AGNs and quasars, of which 672 have spectroscopic redshifts and classifications. Of these, $137(20 \%)$ are type 1 AGNs with blue continua, 294 (44\%) are type 2 objects with extinctions $A_{V} \gtrsim 5$ toward their AGNs, 96 (14\%) are AGNs with lower extinctions $\left(A_{V} \sim 1\right)$, and $145(22 \%)$ have redshifts, but no clear signs of AGN activity in their spectra. Of the survey objects $50 \%$ have $L_{\text {bol }}>10^{12} L_{\odot}$, in the quasar regime. We present composite spectra for type 2 quasars and objects with no signs of AGN activity in their spectra. We also discuss the mid-infrared-emission-line luminosity correlation and present the results of cross correlations with serendipitous X-ray and radio sources. The results show that: (1) obscured objects dominate the overall AGN population, (2) mid-infrared selected AGN candidates exist which lack AGN signatures in their optical spectra but have AGN-like X-ray or radio counterparts, and (3) X-ray and optical classifications of obscured and unobscured AGNs often differ.
\end{abstract}

Key words: galaxies: Seyfert - galaxies: starburst - infrared: galaxies - quasars: general

Online-only material: color figures, machine-readable tables

\section{INTRODUCTION}

The past decade has seen a dramatic improvement in our ability to find active galactic nuclei (AGNs) that would be missing, or strongly selected against, in samples based on selection optical. There are several reasons why finding these objects is of interest. First, the fraction of AGNs obscured by dust represents a significant uncertainty in studies of AGN evolution. Second, the total number of AGNs (obscured plus unobscured) is needed to estimate the mean efficiency of black hole accretion using the Soltan (1982) argument (MartínezSansigre \& Taylor 2009). Third, some obscured AGNs may represent an early phase in AGN activity, as predicted by several models, and finding them would confirm the importance of merger-driven evolution of massive galaxies and give strong clues about the nature of AGN feedback. Fourth, a sample of luminous AGNs without a bright point source nucleus makes host galaxy studies of large numbers of these objects feasible.

Until the Sloan Digital Sky Survey (SDSS) and Spitzer, only a handful of high luminosity obscured AGNs that were neither radio loud (i.e., radio galaxies) nor low luminosity (i.e., Seyfert-2 galaxies, rather than objects of quasar-like luminosity) were known. Norman et al. (2002) found an example radio-quiet type 2 quasar in the Chandra Deep Field South (CDFS), but the small areas of X-ray surveys mean that relatively few high luminosity objects have been discovered in them. Radio-loud examples of this dusty type 1 quasar population had been identified by Webster et al. (1998), but the degree to which synchrotron emission from jets associated with the radio source dominated the optical emission was unclear (Whiting et al. 2001). Using the Two Micron All Sky Survey (2MASS) and near-infrared color selection, Cutri et al. (2002) were able to identify over $200 z \sim 0.2$ radio quiet red AGNs, most likely reddened by dust (a similar technique, but using Spitzer $8 \mu \mathrm{m}$ photometry as the long wavelength data point, was used by Brown et al. 2006). Gregg et al. (2002) used a combination of 2MASS and radio emission in the Faint Images of the Radio Sky at Twenty cm (FIRST) survey to improve the reliability of selection of red quasars by excluding non-AGNs. These techniques typically found less heavily obscured AGNs ( $\left.A_{V} \sim 1-3\right)$, predominately broad-lined objects. This population of lightly dust-reddened AGNs was found to contribute significantly ( $\sim 20 \%)$ to the overall AGN population at quasar-like luminosities, and has properties consistent with an early phase of quasar evolution (Lacy et al. 2002; Glikman et al. 
2004, 2007; Urrutia et al. 2009, 2012). All of these early techniques, however, were still biased against true "type 2" AGNs with rest-frame $A_{V} \gtrsim 10$.

Narrow-line selection of type 2 AGNs from the SDSS (Zakamska et al. 2003; Reyes et al. 2008) increased sample sizes of obscured, high luminosity AGNs to several hundred at $z<0.8$, where the [O III] 5007 line is visible in the optical. Midinfrared selection of obscured AGNs using Spitzer colors was then developed by Lacy et al. (2004), Sajina et al. (2005), and Stern et al. (2005). In parallel, several other groups were working on joint radio/mid-infrared selection techniques (MartínezSansigre et al. 2005, Donley et al. 2005 and Park et al. 2008), which gave more reliable, but less complete, samples, missing radio quiet objects. Mid-infrared techniques have found a significant population of both reddened type 1 and luminous type 2 AGNs at $z>1$ (e.g., Lacy et al. 2007a; hereafter L07), including the discovery of heavily absorbed objects invisible in all but the deepest X-ray surveys. Mid-infrared selected samples are not immune to problems; in particular, they do not discriminate in principle between dust heated by an extremely dense, hot starburst, and an AGN power source (therefore, reliable samples require spectroscopic follow-up, as detailed in this paper) and the technique is not effective at finding AGNs that lack a hot dust component, such as low accretion rate radio galaxies (e.g., Ogle et al. 2006), however, these samples do seem to be complete for rapidly accreting, luminous AGNs.

Using these early mid-infrared samples containing $\sim 10-100$ objects, Martínez-Sansigre et al. (2005) and L07 were able to show that dust-obscured AGNs are at least as common as "normal" blue AGNs, even at high luminosities. These conclusions were also reached by Reyes et al. (2008) using SDSS optical selection of type $2 \mathrm{~s}$, with a much larger sample (887 objects) at $z<0.8$. These samples were, however, too restricted to examine trends of the obscured AGN fraction as a function of both redshift and luminosity. Furthermore, the high redshift objects in the infrared-selected samples are, in general, significantly more luminous than those in X-ray samples of high-redshift AGNs, which are selected from relatively small fields (tens of square arcminutes, compared with tens of square degrees). In these less luminous AGNs, Hubble Space Telescope images tend to reveal little disturbance in the host, even at high redshifts (Kocevski et al. 2012); in contrast, the majority of dust-reddened quasars tend to have host galaxies showing strong signs of disturbance (Lacy et al. 2007b; Urrutia et al. 2008). There, thus may be a difference in the physical causes of AGN activity in Seyfert-like objects powered by secular processes or minor interaction/merger activity in individual galaxies and quasars powered by major galaxy-galaxy mergers (e.g., Treister et al. 2012).

We thus undertook a project to use the Spitzer Wide-area Infrared Extragalactic Survey (SWIRE; Lonsdale et al. 2003), the Spitzer Extragalactic First Look Survey (XFLS; Lacy et al. 2005; Fadda et al. 2006), and the Spitzer Cosmic Evolution Survey (SCOSMOS; Sanders et al. 2007) to search for luminous AGNs in an area of sky totaling $54 \mathrm{deg}^{2}$, large enough to find examples of highly luminous quasars at high redshifts. Furthermore, we nested our survey in terms of flux density limits at $24 \mu \mathrm{m}$ to ensure a good dynamic range in luminosity at a given redshift without the need to take many thousands of spectra. In this paper, we present optical and near-infrared spectroscopy of our new survey. Our paper is structured as follows: Section 2 describes the selection of objects in the survey, how the individual sample flux limits were chosen to ensure a wide range in AGN luminosity at a given redshift, and what selection effects remain. Section 3 describes the spectroscopic observations, Section 4 describes the classification of the objects, Section 5 details the X-ray detections in the survey, Section 6 describes the radio detections, Section 7 discusses the objects lacking AGN signatures in the optical, Section 8 talks about the mid-infrared-emission line luminosity correlation, and Section 9 discusses the composite spectra. Future papers (S. E. Ridgway et al. 2013, in preparation; M. Lacy et al. 2013, in preparation) will discuss the evolution and luminosity dependence of the demographics of the obscured AGN population and the spectral energy distributions (SEDs) of the AGNs.

For the purposes of this paper, we define a quasar as an AGN having a total accretion luminosity of $>10^{12} L_{\odot}(3.8 \times$ $10^{45} \mathrm{erg} \mathrm{s}^{-1} \mathrm{~cm}^{-2}$ ), which, assuming a bolometric correction to $15 \mu \mathrm{m}$ of nine from Richards et al. (2006) for both obscured and unobscured objects, corresponds to $\log _{10}\left(L_{15 \mu \mathrm{m}}\left(\mathrm{erg} \mathrm{s}^{-1} \mathrm{~Hz}^{-1}\right)\right)>31.3$ or $\log _{10}\left(\nu L_{15 \mu \mathrm{m}}\right.$ $\left.\left(\mathrm{erg} \mathrm{s}^{-1}\right)\right)>44.6$. (Note that this result also corresponds approximately to $M_{B}$ (Vega) $<-23.0$ for an unreddened AGN.) We assume a cosmology with $H_{0}=70 \mathrm{~km} \mathrm{~s}^{-1} \mathrm{Mpc}^{-1}, \Omega_{M}=$ 0.3 , and $\Omega_{\Lambda}=0.7$.

\section{SURVEY SELECTION}

\subsection{Infrared Color Selection Criteria}

The candidate AGNs were selected from the XFLS, SWIRE, and SCOSMOS fields. Several samples were selected limited at different $24 \mu \mathrm{m}$ flux density ranges to ensure a good spread of mid-infrared luminosities at a given redshift to aid with disentangling correlations due to redshift from those due to luminosity. Color selection based on the [3.6], [4.5], [5.8], and [8.0] flux densities from the Infrared Array Camera (IRAC) was then applied to each sample (Figure 1) in order to filter out low$z$ starbursts and quiescent galaxies, as detailed in L07 (except with a slightly expanded region in color space allowed for the fainter $24 \mu \mathrm{m}$ samples, as discussed below).

The L07 color "wedge" selection is as follows:

$$
\begin{aligned}
& \log _{10}\left(S_{8.0} / S_{4.5}\right) \leqslant 0.8 \log _{10}\left(S_{5.8} / S_{3.6}\right)+0.5 \\
& \& \log _{10}\left(S_{5.8} / S_{3.6}\right)>-0.1 \\
& \& \log _{10}\left(S_{8.0} / S_{4.5}\right)>-0.2
\end{aligned}
$$

The expanded selection is:

$$
\begin{aligned}
\log _{10}\left(S_{8.0} / S_{4.5}\right) & \leqslant 0.8 \log _{10}\left(S_{5.8} / S_{3.6}\right)+0.5, \\
\& \log _{10}\left(S_{5.8} / S_{3.6}\right) & >-0.3 \\
\& \log _{10}\left(S_{8.0} / S_{4.5}\right) & >-0.3 .
\end{aligned}
$$

The samples are detailed in Table 1. Bright samples (those with flux density limits at $24 \mu \mathrm{m}$ ranging from $4 \mathrm{mJy}$ to $10 \mathrm{mJy}$ ) were selected across all $54 \mathrm{deg}^{2}$ of the SWIRE, XFLS, and SCOSMOS fields in order to maximize the volume probed for very luminous objects. Faint samples, with limits ranging from $S_{24}>0.6$ to $>1.2 \mathrm{mJy}$, were selected in small areas in individual SWIRE fields (totaling $2.2 \mathrm{deg}^{2}$ ) to provide a range in luminosity at every redshift. In addition, we selected a sample with a very narrow range in $S_{24}\left(1.0 \leqslant S_{24}<1.1 \mathrm{mJy}\right)$ split between three separate fields, which we followed up with the Gemini Multi-Object Spectrograph on Gemini South (GMOS-S) to improve our overall spectroscopic completeness at faint $S_{24}$ (the "GMOSS" sample in Table 1). Altogether, our original selection included 963 candidate AGNs (listed 
Table 1

Samples Used

\begin{tabular}{|c|c|c|c|c|c|c|c|c|c|}
\hline Name & $\begin{array}{c}\text { Field } \\
\text { Center(s) }\end{array}$ & $\begin{array}{c}S_{24} \text { Range } \\
(\mathrm{mJy})\end{array}$ & $\begin{array}{l}\text { Effective } \\
\text { Area (sr) }\end{array}$ & $N^{\mathrm{a}}$ & Selection & Follow-up & $\begin{array}{l}\text { Completeness } \\
\left(q^{\mathrm{c}} \leqslant 3\right)(\%)\end{array}$ & $\begin{array}{l}\text { 90\% Flux } \\
\text { Limit }\end{array}$ & $N_{90}^{\mathrm{b}}$ \\
\hline ELAIS-S1 Deep ${ }^{\mathrm{d}}$ & $003444-4328$ & $1.30 \leqslant S_{24}<5.8$ & 0.000103 & 27 & Exp. wedge & CTIO/Hydra & 15 & 2.98 & 4 \\
\hline ELAIS-S1 Bright $^{\mathrm{d}}$ & 003830-4400 & $S_{24} \geqslant 5.8$ & 0.002071 & 37 & Exp. wedge & SOAR/Goodman; Gemini/GMOS & 100 & 6.1 & 36 \\
\hline XMM-LSS Bright $^{\mathrm{d}}$ & 022120-0430 & $S_{24} \geqslant 6.6$ & 0.002772 & 34 & L07 wedge & Hale/COSMIC & 100 & 6.6 & 34 \\
\hline XMM-LSS Deep ${ }^{\mathrm{d}}$ & 021910-0500 & $1.19 \leqslant S_{24}<6.6$ & 0.0000879 & 52 & Exp. wedge & CTIO/Hydra & 33 & 2.35 & 17 \\
\hline CDFS Bright $^{\mathrm{d}}$ & 033200-2816 & $S_{24} \geqslant 6.0$ & 0.002376 & 40 & L07 wedge & SOAR/Goodman; Gemini/GMOS & 100 & 6.0 & 40 \\
\hline GMOS-S ${ }^{\mathrm{d}}$ & $\begin{array}{c}003254-4253, \\
003444-4328, \\
021910-0500\end{array}$ & $1.0 \leqslant S_{24}<1.10$ & 0.000318 & 36 & Exp. wedge & Gemini-S/GMOS; CTIO/Hydra & 81 & $\mathrm{~N} / \mathrm{A}$ & $\mathrm{N} / \mathrm{A}$ \\
\hline SCOSMOS Bright $\mathrm{e}^{\mathrm{e}}$ & $100000+0200$ & $S_{24} \geqslant 6.3$ & 0.0006092 & 8 & L07 wedge & SOAR & 100 & 6.3 & 8 \\
\hline Lockman Bright $^{\mathrm{d}}$ & $104500+5800$ & $S_{24} \geqslant 8.0$ & 0.003351 & 28 & L07 wedge & Hale/COSMIC & 96 & 8.0 & 27 \\
\hline Lockman Deep $^{\mathrm{d}}$ & $105248+5730$ & $1.0 \leqslant S_{24}<8.0$ & 0.0002175 & 133 & Exp. wedge & MMT/Hectospec & 90 & 1.03 & 116 \\
\hline ELAIS-N1 Deep $^{\mathrm{d}}$ & $161024+5436$ & $1.0 \geqslant S_{24}<8.0$ & 0.0002132 & 132 & Exp. wedge & MMT/Hectospec & 86 & 1.306 & 76 \\
\hline ELAIS-N1 Bright $^{\mathrm{d}}$ & $161100+5500$ & $S_{24} \geqslant 8.0$ & 0.002815 & 19 & L07 wedge & Lick/Kast & 88 & 9.6 & 13 \\
\hline ELAIS-N2 Bright ${ }^{\mathrm{d}}$ & $163648+4102$ & $S_{24} \geqslant 8.0$ & 0.001277 & 15 & L07 wedge & Lick/Kast & 100 & 8.0 & 15 \\
\hline XFLS Bright $^{\mathrm{f}}$ & $171800+5930$ & $S_{24} \geqslant 4.6$ & 0.001157 & 46 & L07 wedge & mix; see L07 & 100 & 4.6 & 45 \\
\hline XFLS Deep ${ }^{\mathrm{f}}$ & $171517+5956$ & $0.61 \leqslant S_{24}<4.6$ & 0.0002735 & 178 & Exp. wedge & MMT/Hectospec & 91 & 0.61 & 175 \\
\hline
\end{tabular}

Notes.

${ }^{a}$ Number of objects with either spectroscopy attempted or literature-based redshifts.

b Number of objects in the $90 \%$ complete samples.

${ }^{c}$ Redshift quality (see Section 4.1).

d Spitzer photometry from SWIRE (Lonsdale et al. 2003).

e Spitzer photometry from SCOSMOS (Sanders et al. 2007).

${ }^{f}$ Spitzer photometry from XFLS (Lacy et al. 2005; Fadda et al. 2006).

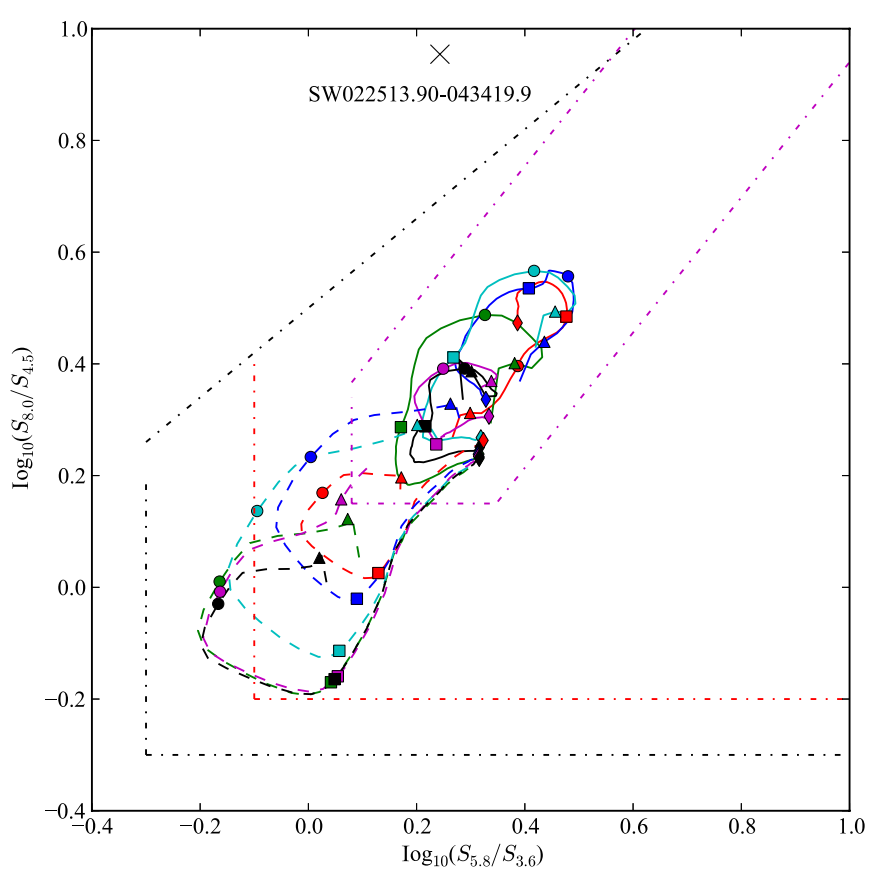

Figure 1. Trajectories of six $z \sim 0.6$ type 2 quasars (from Lacy et al. 2007b, selected to have $S_{[8.0]}>1 \mathrm{mJy}$ ) in color-color space. Solid lines represent the unmodified fits to the SEDs and dashed lines represent the same objects, but with the AGN mid-infrared component reduced by a factor of 10 to represent objects close to the limit of our faintest samples. Symbols represent redshifts of 0.3 (triangles), 1.0 (circles), 2.0 (squares), and 5.0 (diamonds). The red dot-dashed line represents the L07 criteria, the black dot-dashed line represents the expanded wedge used for the faint samples, and the magenta dot-dashed line shows the AGN selection criteria of Donley et al. (2012). The black cross near the top of the plot marks the position of SW022513.90-043419.9, a $z=3.43$ type 2 quasar discovered by Polletta et al. (2008; see Section 6 for a discussion). (A color version of this figure is available in the online journal.) in Table 2); this sample was reduced by constraints on fiber placement to 786 objects for which spectroscopy was attempted or spectroscopic redshifts and type information was provided in the literature (Table 3).

The selection region was changed from the L07 wedge (Equation (1)) to Equation (2) for the fainter samples to improve our completeness in low luminosity AGNs, which tend to have bluer colors as starlight dominates the SED at rest wavelengths $\sim 2-4 \mu \mathrm{m}$ rather than AGN dust emission. The expanded criteria were determined by taking the SEDs of several well-studied type 2 quasars from Lacy et al. (2007b) and changing the redshifts and relative contributions of the AGN components. This method resulted in the trajectories in color space shown in Figure 1 and led us to the conclusion that, to increase completeness, we needed to expand the wedge. Expanding the wedge in this way inevitably results in more contamination, but the spectroscopy is able to remove the objects more likely to be starbursts from the final AGN sample.

Figure 2 shows the actual objects, color coded according to type (see Section 4.2), in the selection region. In general, the AGNs of all types lie close to the locus formed by the type $1 \mathrm{~s}$, however, many of the type 2 s scatter above this line, presumably due to the presence of polycyclic aromatic hydrocarbon (PAH) emission in the mid-IR spectra. There is also a trend for high redshift objects to have redder colors in general. Note that the expanded wedge selection did indeed make the sample more complete to low luminosity type $2 \mathrm{~s}$, although the overall reliability was relatively low within the expansion region-of the 94 extragalactic objects that lie only in the expanded region, $51(54 \%)$ have non-AGN optical spectra, nine (10\%) have featureless spectra, and $34(36 \%)$ have AGN spectra.

Various criteria for the mid-infrared color selection of AGNs are compared and discussed in detail in Donley et al. (2012). 
Table 2

Mid-IR-selected AGN Candidates and Follow-up Spectroscopy Log

\begin{tabular}{|c|c|c|c|c|c|c|c|c|c|}
\hline Object Name & $\begin{array}{c}\text { Telescope/ } \\
\text { Instrument } \\
\text { (or Literature Reference) }\end{array}$ & $\begin{array}{c}\text { Observation } \\
\text { Date (UT) }\end{array}$ & $\begin{array}{l}\text { Exposure } \\
\text { Time } \\
\text { (s) }\end{array}$ & MagSys $^{\mathrm{a}}$ & $m_{2}$ & $m_{4}$ & $m_{5}$ oflg $^{\mathrm{b}}$ & $\operatorname{oref}^{\mathrm{c}}$ & $Z Y J H K$ nflg $^{\mathrm{d}} \operatorname{nref}^{\mathrm{e}}$ \\
\hline SW002644.79-430958.1 ELAIS-S1 Bright & SOAR/Goodman & 2008-08-01UT & 900 & Vega:UBVRI & 19.47 & & 29 & APM & 64 \\
\hline SW002802.79-425957.0 ELAIS-S1 Bright & SOAR/Goodman & 2008-08-03UT & $3 \times 900$ & Vega:UBVRI & 20.28 & & 29 & APM & 64 \\
\hline SW002927.72-431614.4 ELAIS-S1 Bright & Gemini-S/GMOS & 2008-10-10UT & $3 \times 900$ & & & & 64 & & 64 \\
\hline SW002933.86-435240.4 ELAIS-S1 Bright & LF04 & & & Vega:UBVRI & & 17.7 & 23 & LF04 & 64 \\
\hline SW002959.22-434835.1 ELAIS-S1 Bright & LF04 & & & Vega:UBVRI & & 17.42 & 23 & LF04 & 64 \\
\hline
\end{tabular}

Notes. We also include sources that satisfied our selection criteria, but which lacked spectroscopic observations for completeness. Literature references: 2dF: Colless et al. 2001; 6dF: Jones et al. 2009; FBQS: White et al. 2000; LF04: La Franca et al. 2004; Mao12: Mao et al. (2012); P06: Papovich et al. (2006); RC3: de Vaucouleurs, G. et al. 1991; S01: Serjeant et al. 2001; S-A94: di Serego-Alighieri et al. 1994; S06: Simpson et al. 2006; S12: Simpson et al (2012); Sh02: Sharp et al. 2002.

a Optical magnitude system and filters: AB or Vega denotes the system, followed by a colon, then the filter set used in the next five columns $\left(m_{1}-m_{5}\right)$ (i.e., Vega:UBVRI or AB:ugriz). (Note that all the near-IR magnitudes, $Z, Y, J, H$, and $K$, are in the Vega system.)

${ }^{\mathrm{b}}$ Bit flag indicating which optical magnitudes are limits or missing; values are added to the bit flag as follows: $1-m_{1}$ is a limit $/ \mathrm{missing}, 2-m_{2}$ is a limit $/ \mathrm{missing}$, 4- $m_{3}$ is a limit/missing, 8- $m_{4}$ is a limit/missing, 16- $m_{5}$ is a limit/missing. For example, a bit flag of $17(1+16)$ indicates that $m_{1}$ and $m_{5}$ are missing/limits, and the remainder of $m_{2}, m_{3}$, and $m_{4}$ are detections. Where limits are known, they are given, otherwise the magnitude column is left blank. An oflag of 64 indicates that no optical detection has been reported for this object.

c Source of the optical data. APM: Maddox et al. (1990); CASU: Gonzáles-Solares et al. (2011); CFHTLS: Gwyn (2012); COSMOS: Sanders et al. (2007); ESIS: Berta et al. (2006); LCRS: Schechtman et al. 1996; MS4: Burgers \& Hunstead (2006); SDSS: Abazajian et al. 2009; SWIRE: Lonsdale et al. (2003; http://swire.ipac.caltech.edu); USNO-A2: http://ftp.nofs.navy.mil/projects/pmm/catalogs.html). Others as for the spectroscopic references above. "This paper" refers to estimates from spectroscopic acquisition images, or by-eye estimates from available imaging, with an uncertainty $\approx 0.5 \mathrm{mag}$. The other magnitudes are accurate to $\approx 0.1 \mathrm{mag}$ or better; see the respective papers for details. CASU and SWIRE magnitudes are aperture magnitudes in 2.4 or 3.1 arcsec diameters, respectively. The remainder are estimated total magnitudes ("MAG_AUTO" in SEXTRACTOR, Bertin \& Arnouts 1996).

${ }^{\mathrm{d}}$ Bit flag indicating which near-infrared flags are limits or missing; values are added to the bit flag as follows: $1-Z$ is a limit $/ \mathrm{missing}, 2-Y$ is a limit $/ \mathrm{missing}, 4-J$ is a limit/missing, $8-H$ is a limit/missing, $16-K$ is a limit/missing. The resulting nflg is then calculated by summing these values. Where limits are known, they are given, otherwise the magnitude column is left blank. An nflg of 64 indicates that no near-infrared detection has been reported for this object.

e Source of the near-infrared data. 2MASS: Strutskie et al. (2006); DXS, UDS: the Deep Extragalactic Survey and Ultra-Deep Survey of the United Kingdom Infrared Deep Sky Survey (UKIDSS; Lawrence et al. 2007). VIDEO: Jarvis et al. (2013). For DXS and UDS, the 2 arcsec diameter apertures are quoted; for VIDEO, the Petrosian magnitudes. "This paper" refers to estimates from spectroscopic acquisition images, with an uncertainty $\approx 0.5$ mag. The other magnitudes are accurate to $\approx 0.1$ mag or better; see the respective papers for details. Note that all near-infrared magnitudes are in the Vega system.

(This table is available in its entirety in a machine-readable form in the online journal. A portion is shown here for guidance regarding its form and content.)

Table 3

Properties of the AGNs in the Spectroscopic Survey

\begin{tabular}{|c|c|c|c|c|c|c|c|c|c|c|c|}
\hline Object Name & Sample & $\begin{array}{c}\text { In } \\
\text { Statistical } \\
\text { Sample? }\end{array}$ & Redshift & $\begin{array}{l}\text { Redshift } \\
\text { Quality }^{a}\end{array}$ & Type $^{b}$ & $\begin{array}{c}\text { Type } \\
\text { Quality }^{\mathrm{c}}\end{array}$ & $\begin{array}{c}S_{3.6} \\
(\mu \mathrm{Jy})\end{array}$ & $\begin{array}{c}S_{4.5} \\
(\mu \mathrm{Jy})\end{array}$ & $\begin{array}{c}S_{5.8} \\
(\mu \mathrm{Jy})\end{array}$ & $\begin{array}{c}S_{8.0} \\
(\mu \mathrm{Jy})\end{array}$ & $\begin{array}{c}S_{24} \\
(\mu \mathrm{Jy})\end{array}$ \\
\hline SW002644.79-430958.1 & ELAIS-S1 Bright & $\mathrm{Y}$ & 0.241 & 1 & 3 & 2 & 393.79 & 480.71 & 605.92 & 1890.67 & 9629.14 \\
\hline SW002802.79-425957.0 & ELAIS-S1 Bright & $\mathrm{Y}$ & 1.731 & 2 & 4 & 2 & 464.41 & 842.38 & 1655.11 & 3256.47 & 11582.89 \\
\hline SW002927.72-431614.4 & ELAIS-S1 Bright & $\mathrm{Y}$ & 0.593 & 1 & 2 & 1 & 307.22 & 409.86 & 647.29 & 940.93 & 5804.15 \\
\hline SW002933.86-435240.4 & ELAIS-S1 Bright & $\mathrm{Y}$ & 0.994 & 1 & 1 & 1 & 842.44 & 1183.64 & 1701.33 & 2374.71 & 7102.62 \\
\hline SW002959.22-434835.1 & ELAIS-S1 Bright & $\mathrm{Y}$ & 2.039 & 1 & 1 & 1 & 571.40 & 832.07 & 1501.68 & 2611.84 & 6321.39 \\
\hline
\end{tabular}

Notes.Redshift quality, type, and type quality flags are discussed in detail in Sections 4.1 and 4.2 and are summarized here.

${ }^{a}$ Redshift quality: 1: secure redshift based on two or more high signal-to-noise features; 2 : less secure redshift, based on multiple features, but with only one or fewer detected at high signal-to-noise; 3 : uncertain redshift, based on weak spectral features, or a single strong line; 4: featureless spectrum; no redshift estimate.

b Type: 1: normal, unobscured type 1 AGN; 2: heavily obscured, type 2 AGN; 3: no indication of an AGN in the optical spectra; 4: lightly obscured AGN, with broad lines visible in the rest-frame optical, but a red continuum.

${ }^{c}$ Type quality: 1: secure classification (broad lines for type 1s, BPT diagram, [NeV] emission or high-ionization UV lines and a rest-frame optical spectrum for type 2s; clear $(g-i)^{*}$ color excess compared with normal quasars); 2: less secure classification (e.g., only partial information for BPT, low signal-to-noise high-ionization line detections); 3 or 4: uncertain classification due to lack of strong spectral features and/or uncertain line identification.

(This table is available in its entirety in a machine-readable form in the online journal. A portion is shown here for guidance regarding its form and content.)

These authors show that it is possible to come up with better optimized selection for AGNs using IRAC colors, especially with regards to increased reliability of AGN selection. As Figure 2 shows, though, the strictness of the Donley et al. (2012) criteria does result in a significant fraction of AGNs being missed, particularly type 2 AGNs.

\subsection{Biases and Incompletenesses}

We know of several selection effects that will remove objects from these samples. In general, mid-infrared selection does not work well for objects with low accretion rates $(\lesssim 0.01$ Eddington, i.e., low luminosity radio galaxies (Ogle et al. 

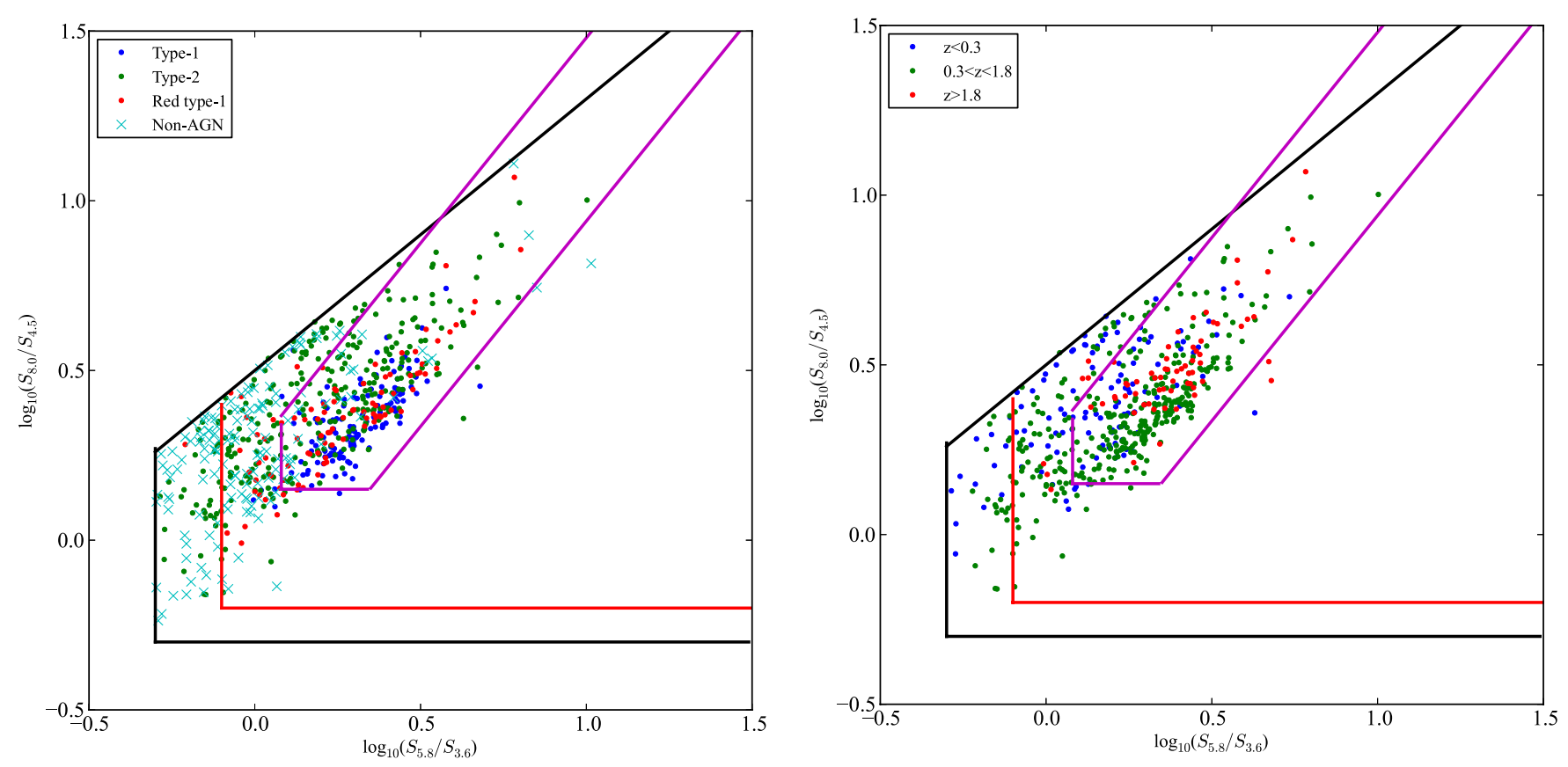

Figure 2. Objects, colored according to (a) type (for all objects) and (b) redshift (for confirmed AGNs only). The selection for the "expanded" wedge, used for the faint samples, is shown as the black solid line. The selection of L07, used for the brighter samples, is shown as the red solid line. The selection criteria of Donley et al. (2012) are shown as the magenta solid line. Note that the Donley et al. criteria also require a monotonic increase in flux from [3.6] through [8.0].

(A color version of this figure is available in the online journal.)

2006) and LINERS (Sturm et al. 2006)), whose mid-infrared luminosities are low compared with their bolometric output, perhaps because they lack the torus structure of objects that accrete at a higher rate, which is thought to be responsible for the mid-infrared emission from AGNs.

A second source of incompleteness was discussed in L07. This incompleteness occurs at $z<0.3$, when the $7.7 \mu \mathrm{m}$ PAH feature from star formation is present in the IRAC $8.0 \mu \mathrm{m}$ band. The PAH feature can be of large enough equivalent width such that the $S_{8.0} / S_{4.5}$ ratio is too high for the object to be classed as an AGN. Objects for which this effect occurs are typically low luminosity objects, with weak mid-infrared continua, since, for a given amount of PAH luminosity, the equivalent width of the feature will be higher if the continuum is weak. However, our overall completeness is significantly compromised at those redshifts (by perhaps as much as 50\%, based on the results of L07). The effects of this incompleteness on our estimate of the AGN luminosity function will be discussed in S. E. Ridgway et al. (2013, in preparation).

A third possible selection effect is related to the highest redshift objects only, where the colors become more difficult to predict and the requirement for detections in the four IRAC bands means that some extreme objects may be missed. Although we have succeeded in finding several high redshift objects, both of the $z \sim 3.5$ objects of Polletta et al. (2008) would have been missed from the color selection due to their very steep mid-infrared spectra at $>5 \mu \mathrm{m}$ in the observed frame (indeed, one of them, SW0225550.32-042149.6, is not even detected at $5.8 \mu \mathrm{m}$ in SWIRE). Objects with extremely low ratios of stellar host luminosity to AGN hot dust luminosity are not represented in our models in Figure 1 (or in Sajina et al. 2005), but seem to be present at high-redshift. The redshift range $z \sim 3-4$ is likely to be particularly problematic, as the $5.8 \mu \mathrm{m} /$ $3.6 \mu \mathrm{m}$ (rest frame $\sim 1.3 \mu \mathrm{m} / 0.8 \mu \mathrm{m}$ ) color is dominated by stellar emission from the host, with only a small contribution from the AGN power law. The color is thus relatively blue, whereas the $8.0 \mu \mathrm{m} / 4.5 \mu \mathrm{m}$ (rest-frame $1.8 \mu \mathrm{m} / 1.0 \mu \mathrm{m}$ ) color can be very red if the AGN is powerful and dominates the $8.0 \mu \mathrm{m}$ flux, as the $4.5 \mu \mathrm{m}$ emission will be dominated by stellar emission from the host. At $z<3$, the effect will be less pronounced. The $5.8 \mu \mathrm{m} / 3.6 \mu \mathrm{m}$ color will be redder because more of the AGN emission is in the $5.8 \mu \mathrm{m}$ band and the object will move into the selection wedge (to the right in Figure 1). Similarly, at $z>4$, the $8.0 \mu \mathrm{m}$ emission from the AGN will be more diluted by the stellar population of the host, rendering the $8.0 \mu \mathrm{m} / 4.5 \mu \mathrm{m}$ color bluer, moving the object down into the selection wedge in Figure 1. A related source of bias against high redshift objects is that high redshift objects with very faint host galaxies would have been missed at the shorter wavelengths, where the host galaxy dominates the SED.

We can quantify some of these incompleteness effects with the aid of the new Spitzer Extragalactic Representative Volume Survey (SERVS; Mauduit et al. 2012), which provides deep IRAC 3.6 and $4.5 \mu \mathrm{m}$ data over a total of $18 \mathrm{deg}^{2}$ spread over several SWIRE fields. First, we examined how many reddened, high-redshift AGNs we may be missing due to their falling outside of our selection wedge (e.g., SW022513.90-043419.9 in Figure 1). In the $4 \mathrm{deg}^{2}$ Lockman Hole SERVS field, we find 38 objects with $S_{24}>1 \mathrm{mJy}$ (comparable to our "Deep" sample flux limits) that have $\log _{10}\left(S_{5.8} / S_{3.6}\right)>0.1$ and lie above the diagonal line in Figure 1 (and therefore are candidates for very high redshift objects missed by our selection wedge). Only 13 of these objects, however, have $r>20$, consistent with their being $z>0.3$ objects missed by our selection. The "Deep" fields in this paper contain 405 objects (with $90 \%$ redshift completeness) within a comparable area $\left(3.2 \mathrm{deg}^{2}\right)$. The addition of $\sim 10$ more high-redshift objects would thus not severely affect the completeness of the sample, although losing these objects may lead to a bias against the highest redshift obscured quasars. Second, we examine how many objects may be missing due to not being detected in the shortest wavelength IRAC bands given the SWIRE and XFLS flux density limits. By combining 
observations with SERVS 3.6 and $4.5 \mu \mathrm{m}$ data, we were able to verify that the faintest object at $3.6 \mu \mathrm{m}$, with $S_{3.6}=8 \mu \mathrm{Jy}$, is above the flux density limit of $\operatorname{SWIRE}\left(S_{3.6}=7.3 \mu \mathrm{Jy}\right)$, although below that of the XFLS $\left(S_{3.6}=20 \mu \mathrm{Jy}\right)$. Given the density of sources with $S_{3.6}<20 \mu \mathrm{Jy}$, we estimate that four objects may be missing from our XFLS Deep sample.

\section{OBSERVATIONS}

\subsection{Optical Spectroscopy}

A wide range of optical facilities and instruments were used for spectroscopic follow-up of our AGN candidates. Most of the bright samples were followed up with 3-5 m telescopes and longslit spectroscopy (Hale with COSMIC, SOAR with Goodman, and Shane with Kast), whereas the fainter samples were followed up with multifiber and/or 6-8 m class telescopes (Blanco with Hydra, MMT with Hectospec, and GeminiSouth with GMOS (program GS-2008B-C4)). We also obtained spectra of some of the bright candidates with a successful poor weather (scheduling band 4) program at Gemini-South (program GS-2008B-Q86). Some objects had spectra available in archives from the SDSS, $2 \mathrm{dF}$, (Colless et al. 2001) or $6 \mathrm{dF}$ (Jones et al. 2009) surveys and some have redshifts and classifications in the literature, all found using the NASA Extragalactic Database. Table 2 (full table in electronic format) gives details of the spectroscopic observations or literature references as appropriate. Note that we include the previously published spectra of L07 for completeness. (For reasons of space, the optical spectra are not shown in this paper, however, we intend to make them available electronically, either by direct application to the first author or through a data service.)

Data analysis followed standard procedures. Most of the data were analyzed in IRAF, using the twodspec package to bias subtract, flat field, wavelength calibrate, and extract. Correction for atmospheric extinction was performed using mean extinction curves appropriate to the observatory. No correction has been made to the spectra for Galactic extinction.

The fiber data from the Hydra instrument were analyzed in the hydra package of IRAF, with improved sky subtraction using our implementation of an algorithm used at the Anglo-Australian Telescope. This algorithm involved median filtering each fiber spectrum with a broad (201 pixel, $168 \AA$ ) filter, subtracting the median-filtered continuum, and then measuring the flux in the prominent sky lines at $5577 \AA, 5892 \AA, 6300 \AA, 6363 \AA$, and $6832 \AA$. The fluxes of the same lines in the combined sky spectrum were then matched to these fluxes and a mean scaling factor was found, which was then applied to the sky spectrum before it was subtracted from the object spectrum.

The MMT Hectospec data were analyzed using software based on the HSRED package of R. Cool, with the same modification discussed above to improve the sky subtraction. In most cases, our sky subtraction technique works well, particularly for the discrete sky lines. Remnant sky lines were masked out in the final spectra. Fiber spectra of faint objects are, however, more vulnerable to artifacts than longslit spectra due to poor sky subtraction. In cases of low signal-to-noise, we therefore attempted to obtain confirmation via near-infrared spectroscopy (Section 3.1) or used SED fitting to check that the redshifts we obtained were consistent with the photometry (see M. Lacy et al. 2013 , in preparation).

In the case of the fiber spectrographs, $\approx 20 \%$ of the candidates did not have spectra taken as we only used a single fiber configuration for each field. In some cases, fibers could not
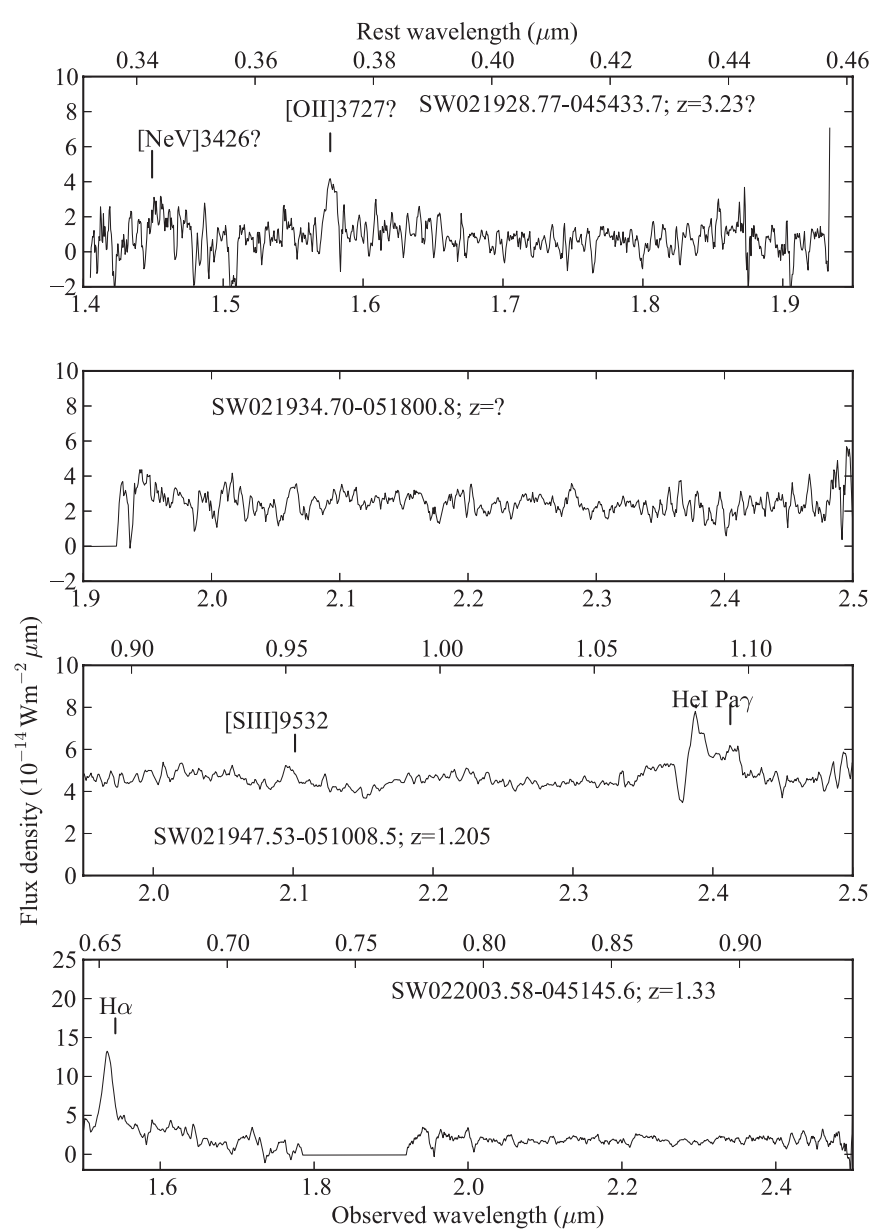

Figure 3. Near-infrared spectra of high-redshift objects in the $X M M$ Deep sample.

be placed close enough together to obtain spectra for all objects (the minimum fiber separation was 20 arcsec for Hectospec and 25 arcsec for Hydra); in other cases, objects were not observed in order to prevent fibers from crossing. For these fields, the effective area was calculated as the area of the survey multiplied by the ratio of the number of objects for which spectra were attempted plus those that had spectra in the literature (and were therefore excluded from the fiber assignments) to the total number of candidates in the area and flux range. Exclusions due to fiber crowding were randomly determined to avoid possible bias.

\subsection{Near-infrared Spectroscopy}

For some high-redshift candidates with ambiguous or low signal-to-noise optical spectra, we were able to obtain near-infrared spectra with the IRTF using SpeX (Rayner et al. 2003), Gemini with NIRI (program GN2009B-C-8), and Triplespec (Herter et al. 2008) on Palomar. The near-infrared observations are listed in Table 4 and the corresponding spectra are shown in Figures 3-6.

\section{REDSHIFTS AND SPECTRAL CLASSIFICATIONS}

\subsection{Redshift Completeness and Quality Flags}

Redshifts and classifications for the objects are shown in Table 3. Objects were assigned a redshift quality flag from 1 to 4 according to the following criteria. 
Table 4

Near-infrared Spectroscopy

\begin{tabular}{|c|c|c|c|c|c|}
\hline Object & Subsample & Telescope & Instrument/Band & Integration Time & $\begin{array}{l}\text { Observation Date } \\
\text { (yyyy-mm-dd) }\end{array}$ \\
\hline SW $021928.77-045433.7$ & XMM-LSS Deep & Gemini-N & $\mathrm{NIRI/H}$ & $37 \times 120$ & $2009-11-29$ \\
\hline SW 021934.70-051800.8 & XMM-LSS Deep & Gemini-N & NIRI/K & $37 \times 120$ & $2009-11-28$ \\
\hline SW 021947.53-051008.5 & XMM-LSS Deep & Gemini-N & NIRI/K & $13 \times 120,18 \times 120$ & 2009-12-08, 09 \\
\hline SW $022003.58-045145.6$ & XMM-LSS Deep & Gemini-N & NIRI/J, K & $31 \times 120,37 \times 120$ & $2009-11-29,2009-11-30$ \\
\hline SW $022003.58-045145.6$ & XMM-LSS Deep & Hale & Triplespec & $8 \times 300$ & 2008-07-27 \\
\hline SW 104839.73+555356.4 & Lockman Bright & Gemini-N & NIRI/K & $26 \times 120$ & $2009-11-29$ \\
\hline SW 105201.92+574051.5 & Lockman Deep & Gemini-N & $\mathrm{NIRI} / \mathrm{K}$ & $25 \times 120$ & $2009-11-28$ \\
\hline SW 105213.39+571605.0 & Lockman Deep & Gemini-N & NIRI/K & $19 \times 120$ & $2009-11-28$ \\
\hline SW 160913.28+542322.0 & Lockman Deep & Hale & Triplespec & $20 \times 300$ & 2011-07-12 \\
\hline SW 163313.28+401338.9 & EN2 Bright & Hale & Triplespec & $12 \times 300$ & 2011-07-11 \\
\hline XFLS $171053.51+594433.1$ & XFLS Faint & Hale & Triplespec & $24 \times 300$ & 2011-07-11 \\
\hline XFLS 171419.9+602724 & XFLS Bright & IRTF & Spex & $30 \times 120$ & 2007-06-18 \\
\hline XFLS $171503.96+595959.3$ & XFLS Faint & Hale & Triplespec & $24 \times 300$ & 2011-07-12 \\
\hline XFLS 171702.54+600620.7 & XFLS Faint & Hale & Triplespec & $16 \times 300$ & 2011-07-11 \\
\hline XFLS 171754.6+600913 & XFLS Bright & IRTF & Spex & $30 \times 120$ & 2007-06-19 \\
\hline
\end{tabular}

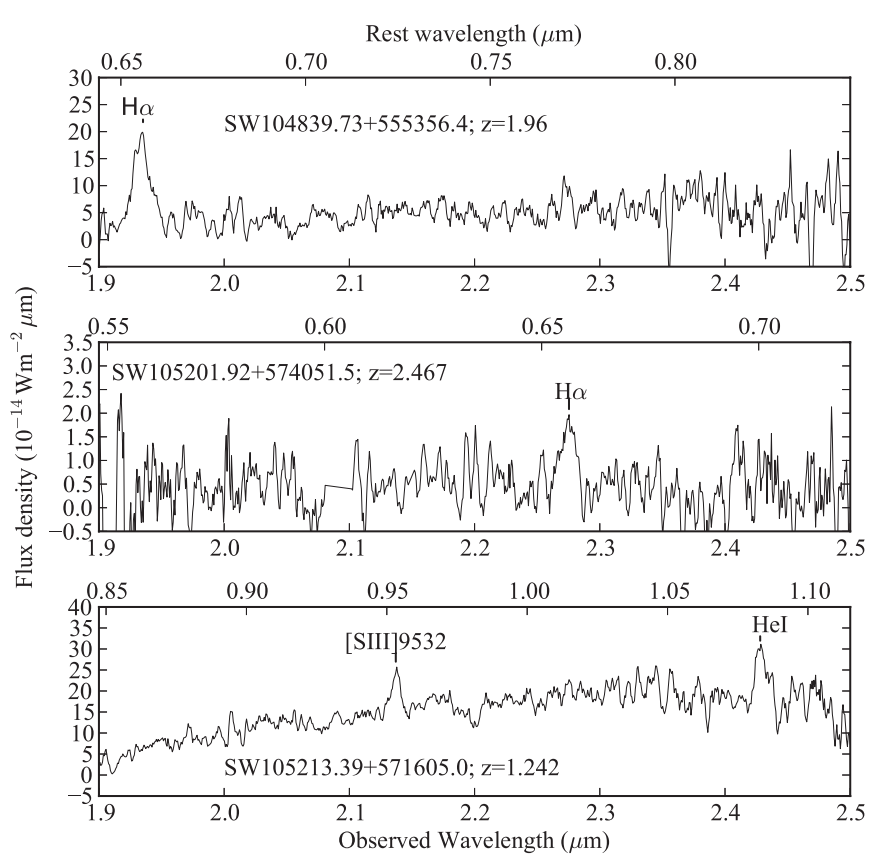

Figure 4. Near-infrared spectra of high-redshift objects in the Lockman Bright and Lockman Deep samples.

1. Secure redshift based on a high signal-to-noise $(>10 \sigma)$ detection of two or more spectral features (in emission or absorption).

2. Less secure redshift, based on the detection of one emission line at high signal-to-noise plus one line at lower signal-to-noise $(>3 \sigma)$, or more than two lines at lower signal-to-noise.

3. Uncertain redshift based on a single weak line with only weak secondary features to obtain the redshift, or a single strong line whose identity was deduced from its profile or the absence of other lines expected to be similarly strong in the spectrum.

4. Featureless spectrum, or spectrum with only a single weak line and no other features (no redshift assignment attempted).

Objects with redshift quality 2 or 3 were also checked for the likelihood of the assumed redshift using SED fitting using

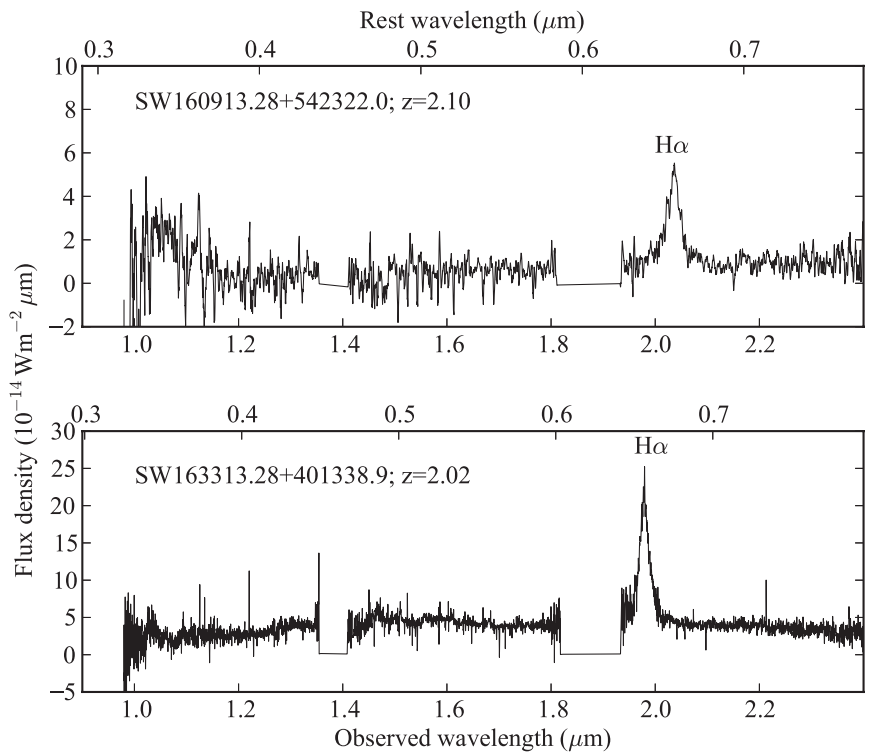

Figure 5. Near-infrared spectra of high-redshift objects in the ELAIS-N1 Bright and ELAIS-N2 Bright samples.

models similar to those in Lacy et al. (2007b). In cases that these redshifts led to unphysical SEDs (for example, host galaxy masses $\gg 10^{12} M_{\odot}$ or significant dust emission corresponding to dust temperatures above the sublimation temperature of $\approx 1500 \mathrm{~K})$, the redshifts were assumed to be incorrect and the redshift quality was set to 4 . The full details of the SED fitting will be given in M. Lacy et al. (2013, in preparation). Figure 7 shows the distribution of redshift quality by object classification (see Section 4.2). As expected, the unreddened AGNs have the lowest fraction of poor quality redshifts. The redshift distribution of the non-AGNs cuts off abruptly at $z \approx 1$, probably because higher redshift objects in this class lack strong emission features in their rest-frame UV spectra and therefore would appear to have featureless spectra.

The overall redshift completeness in the samples varied from $100 \%$ for most of the bright samples to $\sim 20 \%$ for some of the faint samples. In order to obtain a subset of the survey useful for statistical purposes in S. E. Ridgway et al. (2013, in preparation), $a \geqslant 90 \%$ complete subsample was computed for each individual sample by exploiting the correlation of $S_{24}$ with 

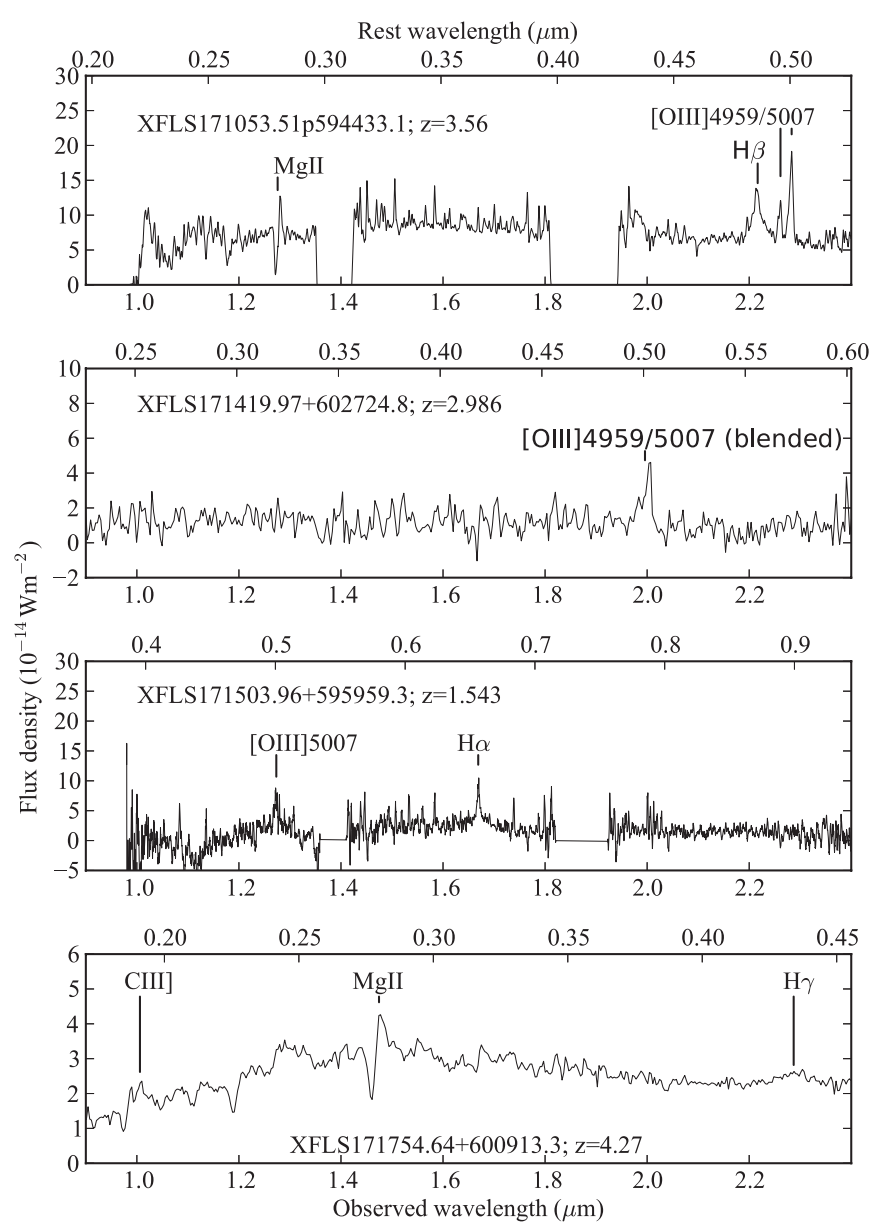

Figure 6. Near-infrared spectra of high-redshift objects in the XFLS Bright and XFLS Faint samples.

emission-line flux (except for the GMOS-S data, which have a very narrow range in $S_{24}$ ). This procedure was carried out as follows: each sample was sorted in descending order of $S_{24}$. The completeness was then calculated as a function of $S_{24}$ (assuming all redshifts with qualities 1-3 were correct). Objects were included in the $90 \%$ complete sub-sample until the completeness as a function of $S_{24}$ dropped below $90 \%$ for the final time (see Table 1). These $90 \%$ complete samples, combined with the $81 \%$ complete, GMOS-S sample, constitute the "statistical sample" of 662 objects.

The histogram of redshifts broken up by type (as described below), together with the $24 \mu \mathrm{m}$ flux distribution of the objects lacking redshifts, are shown in Figure 8. As expected, most of the objects lacking redshifts are faint at $24 \mu \mathrm{m}$ (see Section 8). There does seem to be a deficit of type 2 objects at $1.4<z<$ 1.6, analogous to the traditional "redshift desert" for normal galaxies, when [O II] 3727 redshifts out of the optical band and before Ly $\alpha$ redshifts in (see also the top-right panel of Figure 7). For our type 2 objects, the C IV 1549 line is typically brighter than Ly $\alpha$, which we believe is why the redshift desert for our objects extends only to $z \approx 1.6$, where C IV is shifted to $4000 \AA$, the blue end of most of our spectra. The type 1 objects are not affected by this situation, as they have strong low-ionization broad lines (Mg II 2798 and C III]1909) in the observed optical bands at these redshifts. These low-ionization UV lines tend to be relatively weak in the type 2 s (see Section 9). The deficit may also be in part due to the fact that samples that are flux limited at $24 \mu \mathrm{m}$ containing heavily obscured objects are biased against these redshifts, as the $9.7 \mu \mathrm{m}$ silicate feature is redshifted into the $24 \mu \mathrm{m}$ band in this range.

\subsection{Classification}

Objects were classified as either type 1 (normal, unobscured; type $=1$ in Table 3 ), type 2 (heavily obscured; type $=2$ in Table 3), lightly obscured, with broad lines visible in the rest-frame optical, but a red continuum (type $=4$ in Table 3 ), or showing no evidence for an AGN in their optical spectra (type = 3 in Table 3; called "non-AGN" in the text of this paper, although some of them in fact are AGNs based on other criteria; see Section 7). There are also 24 stars, most likely contaminants due to saturated IRAC flux densities (although probably possessing debris disks to be bright at $24 \mu \mathrm{m}$ ), classified as type $=5$. The criteria followed those employed by L07, based principally on emission-line properties (presence of broad lines, classification in the BPT diagram, presence of $[\mathrm{NeV}]$ emission, or presence of high ionization UV lines in emission), although a more refined definition of dust-reddened versus normal AGNs was used, as described below. Where possible, a rest-frame optical spectrum was used to distinguish between a type 2 AGN and a lightly obscured AGN. Objects with uncertain types have type quality 2 or greater. Typically, these are objects that lack a full set of detected lines to plot on a diagnostic diagram or are high-redshift objects without a rest-frame optical spectrum.

We can be fairly sure that objects showing narrow, high ionization UV emission lines are AGNs based on comparison with radio galaxies and X-ray detected type 2 quasars (e.g., Norman et al. 2002). Although high ionization resonant emission lines such as Si IV $1402 \AA$ and CIV1548/1551 $\AA$ can be formed in starbursts, in the stellar winds and photospheres of the most massive stars, as well as in the interstellar medium (e.g., Robert et al. 1993), these lines are predicted to form P-Cygni profiles, with substantial absorption from gas blueshifted along the line of sight, which we do not see in our objects. Furthermore, in practice, Lyman break galaxies show high-ionization species predominately in absorption (Shapley et al. 2003). High ionization species that do appear in emission, such as He II $1640 \AA$ and $\mathrm{C}$ III] $1909 \AA$, tend to have very low rest-frame equivalent widths $(\lesssim 2 \AA)$. We also have several known examples of objects that show narrow lines in the rest-frame UV, but broad lines in their near-infrared, rest-frame optical spectra (e.g., XFLS 171053.51+594433.1), which reinforce the point that near-infrared spectra are needed for accurate classification of a type 2 objects versus a reddened type 1 object. For objects that lack near-infrared spectroscopy, we have been able to make an approximate classification based on SED shapes, however, these classifications are uncertain and have type quality $=2$ or 3 .

A significant fraction of our mid-infrared selected AGNs have broad-line spectra, but are clearly redder than the optically selected quasar population (the objects classified as type $=4$ in Table 3). This red AGN population has also been found in other surveys based on selection from 2MASS (e.g., Cutri et al. 2003) or 2MASS and the FIRST radio survey (e.g., Glikman et al. 2004; 2007; Urrutia et al. 2009). Classification of red versus normal AGNs needs to take into account (at least in a statistical sense) the intrinsic variation in AGN colors and be empirically based, so as not to be tied to a specific reddening law. We have adopted the technique of Richards et al. (2003), who use the $g-i$ color excess relative to the mean for SDSS quasars as a measure of reddening. These authors argue that intrinsic color variations are likely to be symmetrically distributed around 

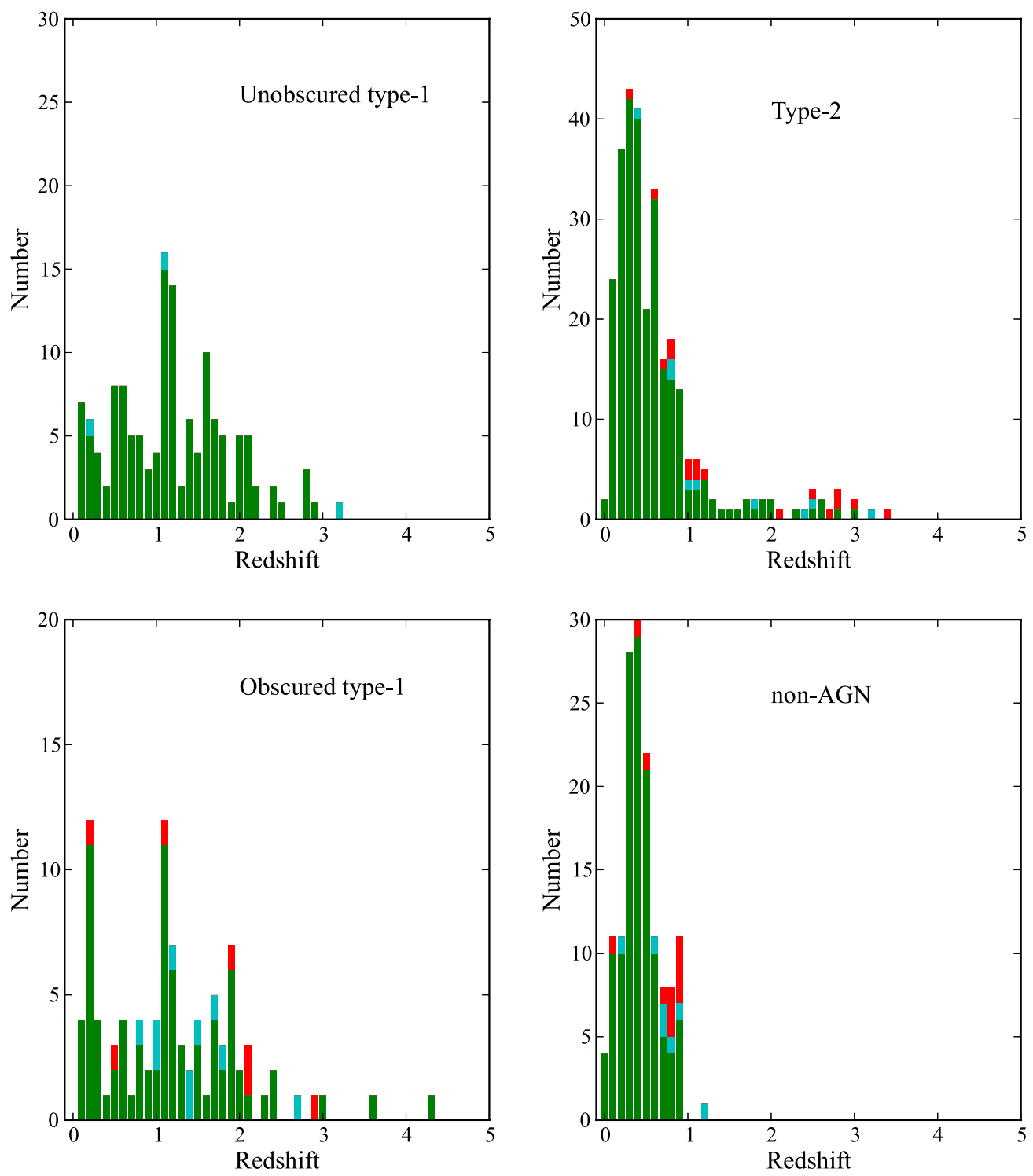

Figure 7. Histograms of redshift for each of the four classes of objects. Objects with redshift quality 1 are shown in green, those with redshift quality 2 are shown in blue, and those with redshift quality 3 are shown in red.

(A color version of this figure is available in the online journal.)

the mean color and that the objects with red excesses beyond this distribution are likely to be reddened by dust. Most of our objects have photometry in the SDSS system and thus we are able to use the $(g-i)$ reference colors as a function of redshift from Richards et al. to calculate the distribution of excess $(g-i)$ color, $(g-i)^{*}$, for 193 of our objects that are classified as type 1 (normal or reddened) and have photometry in the SDSS system (samples XMM-LSS Bright, XMM-LSS Deep, Lockman Bright, Lockman Deep, EN1 Bright, EN1 Deep, EN2 Bright, XFLS Bright, and XFLS Deep), shown in Figure 9 (left panel). The peak around zero reddening has a standard deviation (after clipping) of $\approx 0.15 \mathrm{mag}$. We therefore place our empirical boundary between reddened and unreddened objects two standard deviations away, at $(g-i)^{*}=0.3$. This adoption of an observed-frame color to distinguish red from normal AGNs means that the value of reddening in the rest-frame that defines the boundary between reddened and unreddened AGNs will vary as a function of redshift (as a given optical depth of dust will result in a higher $(g-i)^{*}$ in high-redshift objects), however, only a handful of objects have colors close enough to the boundary to make their classifications uncertain due to this fact. The restframe $E(B-V)$ obtained from fitting a reddened template quasar SED to SEDs of objects close to our proposed boundary implies that a $(g-i) *$ color excess of 0.3 mag corresponds to an $E(B-V) \approx 0.1-0.15$ at $z \sim 1$, previously used by us to define red quasars (e.g., Urrutia et al. 2009). The 24 objects without colors in the SDSS system (objects in the GMOS-S, CDFS Bright, ES1 Bright, and ES1 Deep samples) were evaluated on the basis of available optical colors as close to $g$ and $i$ as possible. In most cases, classification was unambiguous, however, in five cases that were close to the estimated boundary a classification of unreddened was adopted.

We also used the SED of Richards et al. (2006) to calculate the $r-$ [24] color excess between the optical and mid-infrared, $(r-[24])^{*}$ (Figure 9 (right panel)). There is a clump of normal AGNs near $(0,0)$, consistent with the idea that we are dominated by the normal AGN population and are not systematically selecting objects with unusually bright infrared emission. 

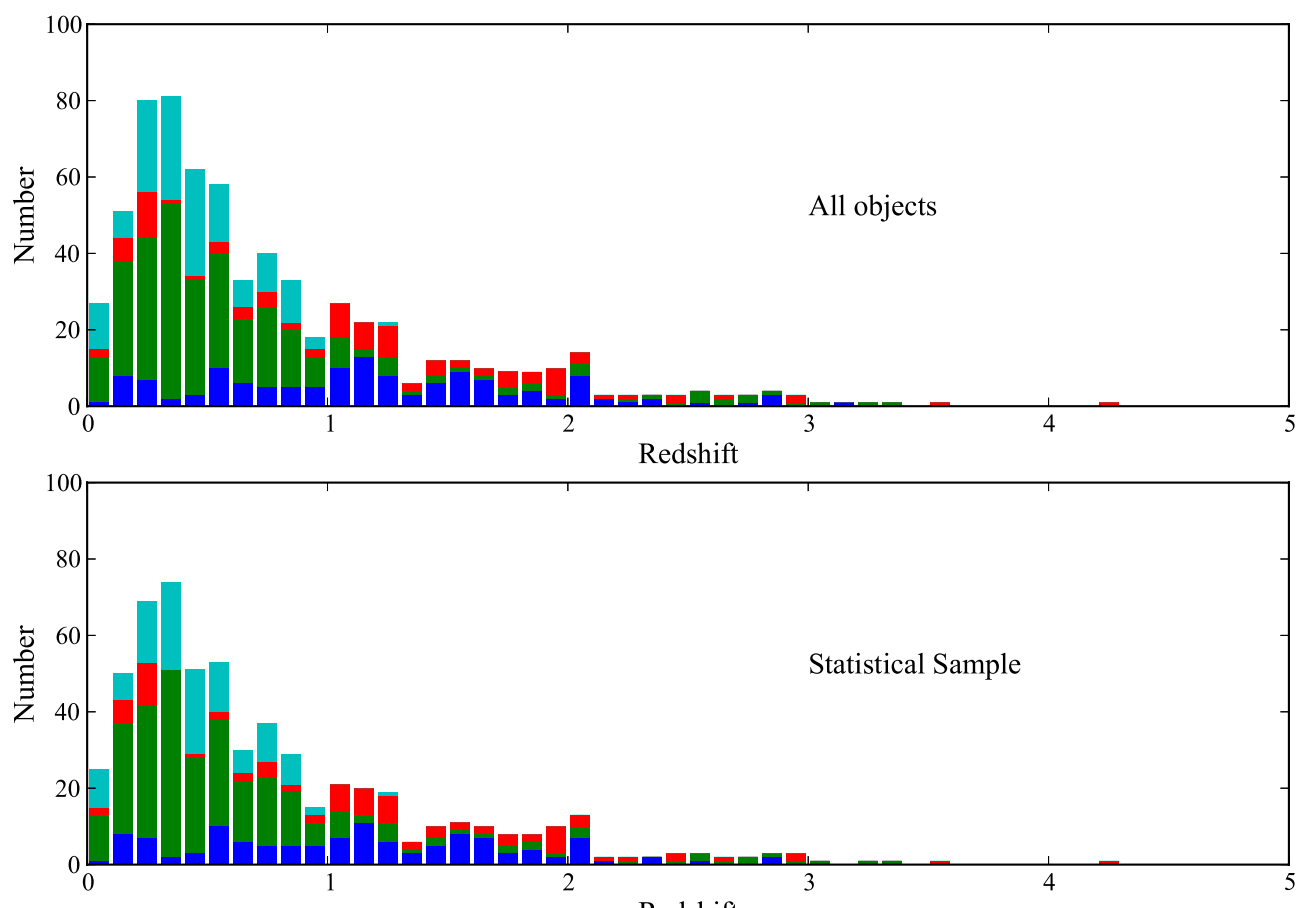

Redshift

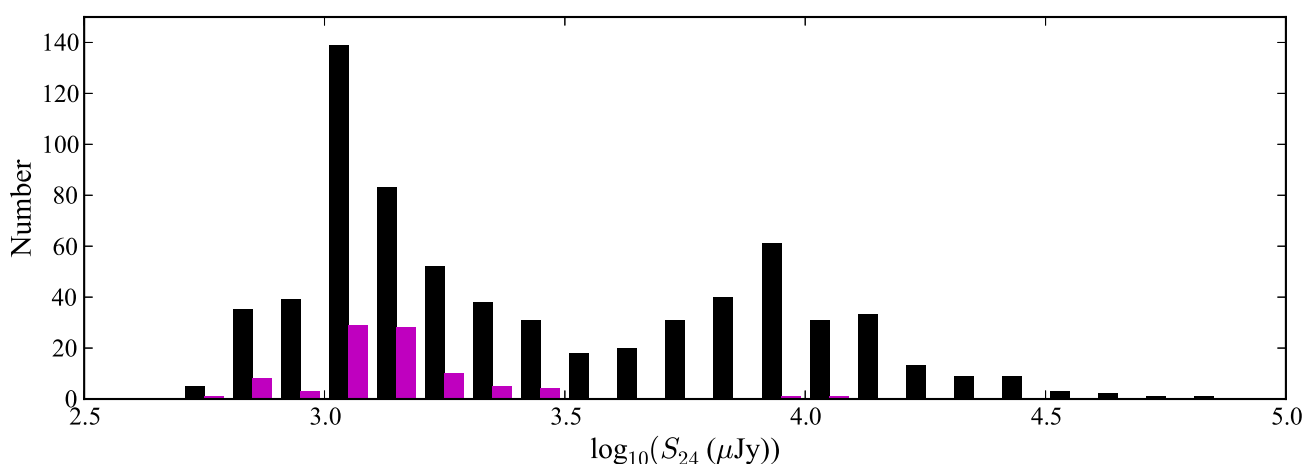

Figure 8. Histograms of redshifts by object type: type 1 objects are shown in blue, type 2 objects are shown in green, reddened type 1 objects are shown in red, and objects with non-AGN optical spectra are shown in cyan. The bottom histogram shows the $24 \mu \mathrm{m}$ flux distribution for the objects with featureless spectra (magenta) compared with the remainder of the sample (black).

(A color version of this figure is available in the online journal.)
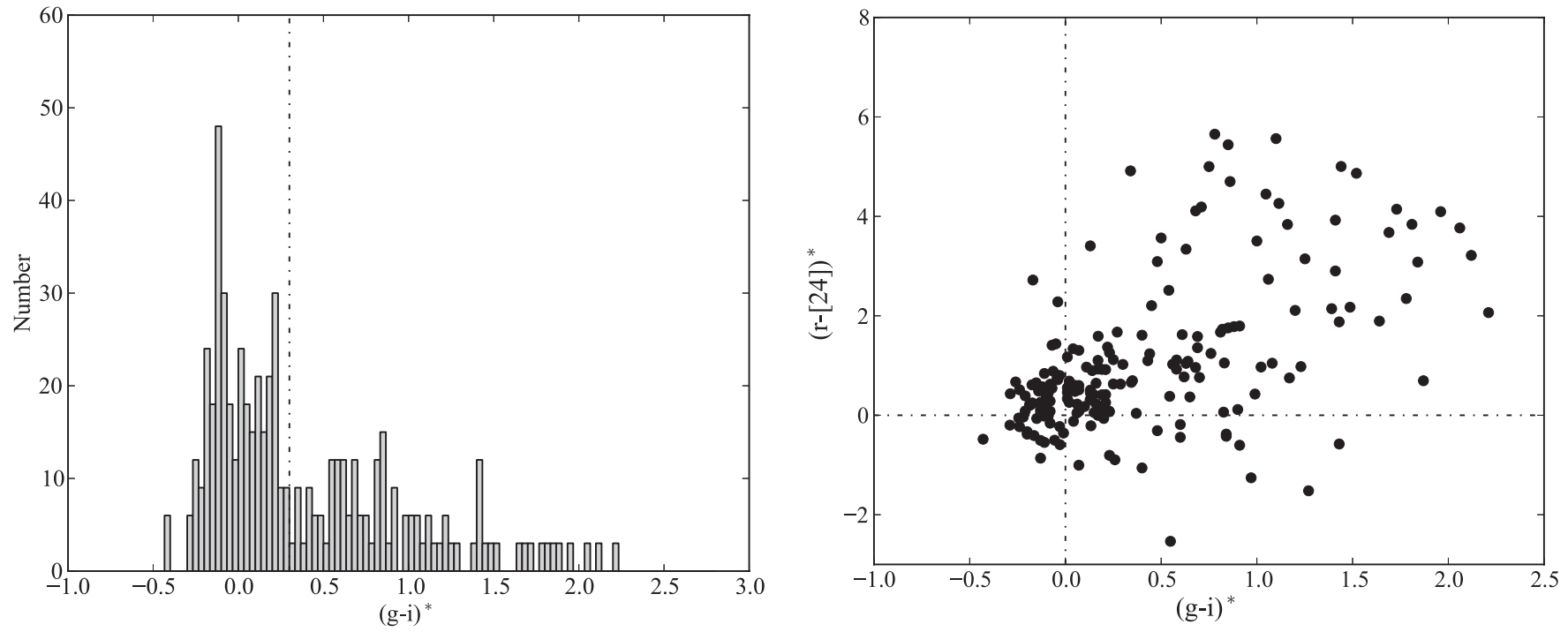

Figure 9. Left: histogram of corrected $g-i$ color for the objects classified as type 1 (i.e., having broad lines in the rest-frame optical). Right: corrected $r-$ [24] color plotted against excess $g-i$ color for the broad-line objects. 
As expected for dust reddening, $(g-i)^{*}$ correlates broadly with $(r-[24])^{*}$, although the correlation is surprisingly poor. The objects scattered toward bluer $(r-[24])^{*}$ colors seem to be explained in terms of host galaxy contamination, as the majority of objects in this area of the plot are at low redshift and show evidence of host galaxy starlight in their spectra. Conversely, the outliers with high $(r-[24])^{*}$ and low $(g-i)^{*}$ tend to be very faint in the optical, where again the host galaxy emission and (possibly) scattered AGN light may make the observed colors bluer. The lack of a tight correlation for the more highly reddened objects (redder than $(g-i)^{*}=0.3$ ) may also reflect genuine variation in the ratio of reddening (measured by $\left.(g-i)^{*}\right)$ to optical extinction (approximated by $\left.(r-[24])^{*}\right)$ in our population.

\section{X-RAY DETECTIONS}

We matched the objects in our spectroscopic survey in Table 3 to the 2XMM-DR3 catalog of serendipitous XMM sources (Watson et al. 2009) and the Chandra Source Catalog (CSC; Evans et al. 2010), finding 108 matches to the 2XMM and 81 to the CSC (36 of which are in both catalogs, Table 5). Both catalogs have only patchy coverage across the survey fields and exposure times ranging from $\sim 5-50 \mathrm{ks}$, so the low detection fraction is no surprise. As pointed out in L07 and Donley et al. (2012), however, many obscured AGNs are not detected even in deep X-ray surveys. The breakdown, among the X-ray-detected sources by type, is 52 normal type $1 \mathrm{~s}, 45$ type $2 \mathrm{~s}, 31$ red type $1 \mathrm{~s}, 9$ objects with redshifts but no optical AGN signatures, 14 objects with featureless spectra, and 2 stars. Four of the 2XMM sources and one of the CSC sources are weaker than $10^{42} \mathrm{erg} \mathrm{s}^{-1} \mathrm{~cm}^{-2}$, where their X-ray luminosity could plausibly be powered by $\mathrm{X}$-ray binaries in the host galaxy (e.g., Teng et al. 2005), but the vast majority are much more luminous and are almost certainly powered by AGNs.

Figure 10 shows the X-ray flux against the $24 \mu \mathrm{m}$ flux, a histogram of the X-ray to $24 \mu \mathrm{m}$ flux ratio, a distribution of hardness ratio (HR) by type (for $X M M$, we use $H R 2$, defined to be the HR between $0.5-1 \mathrm{keV}$ and 1-2 keV; for Chandra, we use the HR defined between the "hard" and "soft" ACIS bands, called $H R C$ in this paper), and the X-ray luminosity versus the mid-infrared luminosity. The mid-infrared $k$-correction is calculated using the measured 8-24 $\mu \mathrm{m}$ spectral index. For both the $2 \mathrm{XMM}$ and CSC detections, the type 1 objects are generally the brightest and softest in X-rays $(\langle H R C\rangle=-0.13 \pm 0.25$ and $\langle H R 2\rangle=0.02 \pm 0.25)$ and their X-ray fluxes correlate loosely with their $24 \mu \mathrm{m}$ fluxes. The type $2 \mathrm{~s}$ and other obscured populations are, on average, the faintest and have a wide range of HRs $(\langle H R C\rangle=0.14 \pm 0.37 ;\langle H R 2\rangle=0.15 \pm 0.48$ for type $2 \mathrm{~s},\langle H R 2\rangle=-0.04 \pm 0.31 ;\langle H R 2\rangle=0.31 \pm 0.35$ for red type $1 \mathrm{~s},\langle H R 2\rangle=-0.14 \pm 0.22 ;\langle H R 2\rangle=0.45 \pm 0.53$ for optically classified "non-AGNs," and $\langle H R 2\rangle=0.21 \pm 0.34$; $\langle H R 2\rangle=0.40 \pm 0.37$ for objects with featureless optical spectra. If we were to reclassify objects purely on the basis of their $\mathrm{X}$-ray HRs and assume a division of $H R C=0$ or $H R 2=0.1$ as the boundary of "obscured" versus "unobscured," $19 \%$ $(12 / 62)$ of the optically classified unobscured type 1 objects would be classified as obscured in the X-ray. Conversely, $41 \%$ $(24 / 59)$ of the type 2 objects would have been classified as X-ray unobscured, along with $51 \%(19 / 37)$ of the reddened type 1 objects, $50 \%(5 / 10)$ of the objects with non-AGN spectra, and $26 \%(7 / 12)$ of the objects with featureless spectra. These differences are, however, subject to systematic uncertainties as we only consider X-ray detected objects and the obscured objects in particular tend to be faint, rendering their HRs uncertain, and subject to contamination by soft emission from scattering of AGN emission or X-ray binaries in the hosts. Nevertheless, our results are consistent with those of Brightman \& Nandra (2011), who also note discrepancies between X-ray and optical classifications of AGNs. On the X-ray luminosity-midinfrared luminosity plots, the correlation for the $2 \mathrm{XMM}$ points (whose fluxes are more weighted toward the soft X-rays) is weaker than for the $2-10 \mathrm{keV}$ Chandra luminosities.

\section{RADIO DETECTIONS}

The fields used for this survey overlap with several moderate depth (root mean square (rms) sensitivity $\approx 10-30 \mu \mathrm{Jy}$ ) radio surveys at $1.4 \mathrm{GHz}$. In the north, the XFLS Bright and XFLS Deep samples overlap with the Very Large Array (VLA) survey of Condon et al. (2003), the Lockman Deep sample overlaps with the Ibar et al. (2009) VLA survey, and the ELAIS-N1 Deep sample overlaps with the VLA survey of Ciliegi et al. (1999). In the south, the ELAIS-S1 Bright, ELAIS-S1 Deep, and CDFS Bright surveys overlap the Deep Australia Telescope Large Area Survey made with the Australia Telescope Compact Array (Norris et al. 2006; Middelberg et al. 2008). We have crossmatched our survey AGNs with the public source lists from these surveys in order to make an initial examination of the radio properties of our objects, detecting 214 objects. In the XFLS Bright and XFLS Deep fields, which overlap the deep (rms $10 \mu \mathrm{Jy}$ ) and uniform radio survey of Condon et al., our detection rate is $90 / 223$, or $40 \%$, so even in the fields with the best radio data the majority of objects retain upper limits.

Figure 11 shows the flux-flux plot, the luminosity-luminosity plot, and a histogram of radio loudness, where we define the $k$ corrected infrared radio-loudness as

$$
R_{I R}^{*}=\log _{10}\left(F_{5 \mathrm{GHz}} / F_{15 \mu \mathrm{m}}\right),
$$

where $F_{5 \mathrm{GHz}}$ and $F_{15 \mu \mathrm{m}}$ are the rest-frame fluxes at $5 \mathrm{GHz}$ and $15 \mu \mathrm{m}$, respectively. A radio spectral index $\alpha=-0.8$ (where flux density $S_{v} \propto v^{\alpha}$ ) was assumed. We also show the approximate position of the far-infrared-radio correlation adapted from Appleton et al. (2004) with a small ( -0.2 dex) correction to allow for the different infrared and radio wavelengths used.

Most AGNs lie close to the far-infrared-radio correlation, even when the mid-infrared, which is dominated by AGN luminosity, is used, as has been noted in the past (Sopp \& Alexander 1991; Kimball et al. 2011). We also see no sign of any radio-loudness dichotomy or bimodality in any of our object classifications, consistent with most recent studies (White et al. 2007; Kimball et al. 2011). We do, however, see significant scatter both above the far-infrared-radio relation (the expected radio-loud/intermediate population) and below (where objects with strong AGN-related mid-infrared emission appear). Furthermore, the distribution of $R_{I R}^{*}$ values for the broad-line objects (with a mean of -1.35 with an error in the mean of $\approx 0.1$ for both the normal and obscured type 1 populations) is lower than that for the type 2 population $(-1.09 \pm 0.05)$ and the nonAGNs $(-1.05 \pm 0.05)$. We performed both a Mann-Whitney $U$-test and a $t$-test to investigate whether the values of $R_{I R}^{*}$ are systematically lower for the broad-line objects taken as a group versus the type $2 \mathrm{~s}$, finding a $3 \%$ probability of the samples being drawn from identical parent populations in both cases. Thus, the difference is not highly robust and, furthermore, the large number of upper limits may mean that the result may be biased by the different redshift and luminosity distribution of the 

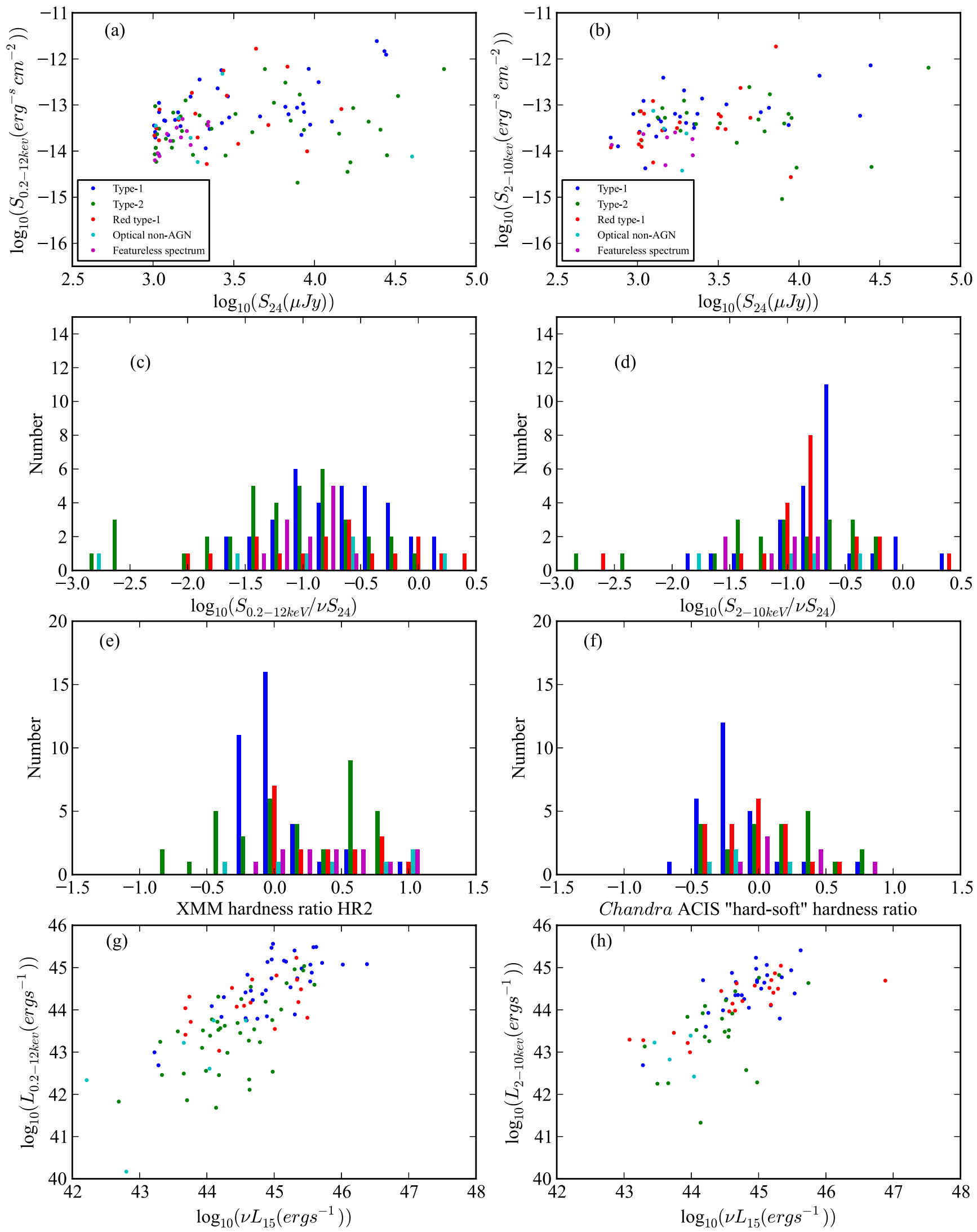

Figure 10. X-ray detections and HRs of objects in the survey by type. (a) XMM $0.2-12 \mathrm{keV}$ flux vs. $24 \mu \mathrm{m}$ flux density, (b) Chandra $2-10 \mathrm{keV}$ flux vs. $24 \mu \mathrm{m}$ flux density, (c) ratio of XMM 0.2-12 keV to $24 \mu \mathrm{m}$ flux, (d) ratio of Chandra 2-10 keV flux to $24 \mu \mathrm{m}$ flux, (e) distribution of XMM hardness ratio HR2, (f) distribution of Chandra hardness ratio, (g) XMM luminosity vs. $15 \mu \mathrm{m}$ luminosity, and (h) Chandra luminosity vs. $15 \mu \mathrm{m}$ luminosity. The color coding of the histograms is given in the top panels.

(A color version of this figure is available in the online journal.) 

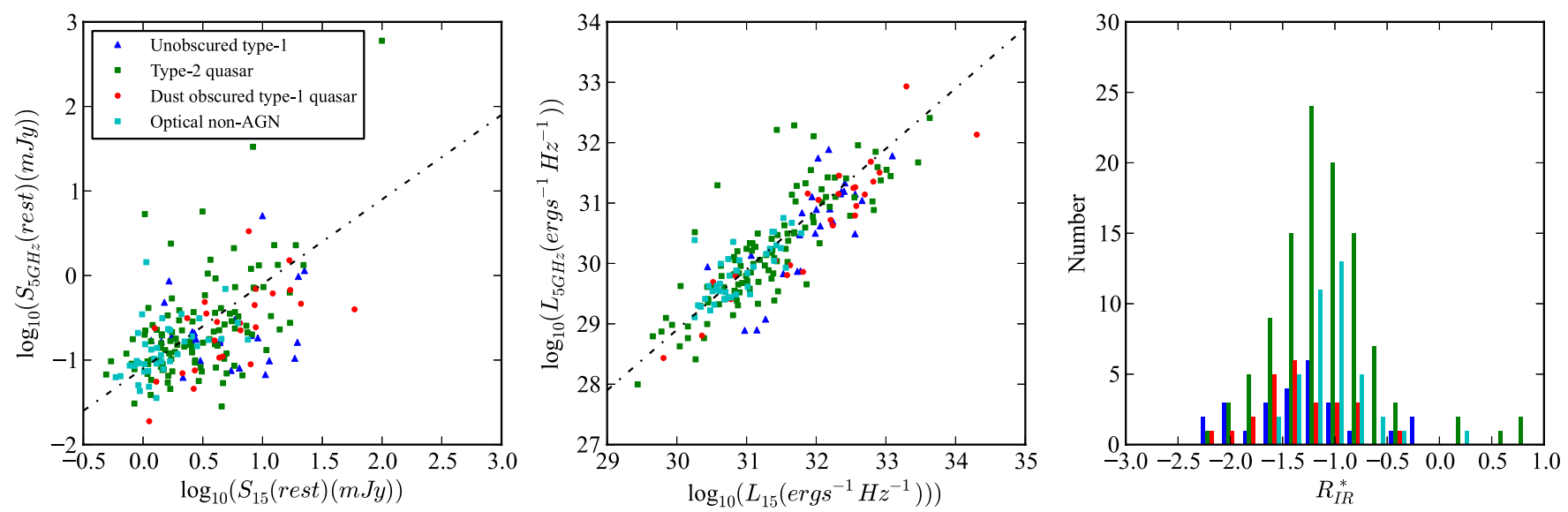

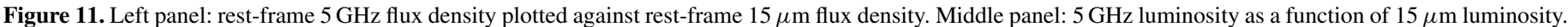

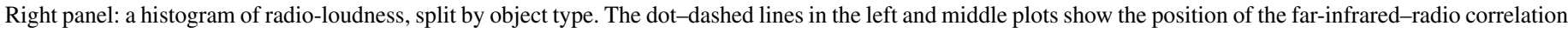
from Appleton et al. (2004), scaled to $15 \mu \mathrm{m}$ and $5 \mathrm{GHz}$, assuming a mid-infrared spectrum flat in $v f_{v}$ and a radio spectral index of -0.8 .

(A color version of this figure is available in the online journal.)

Table 5

X-Ray Properties of AGNs in the Spectroscopic Survey

\begin{tabular}{|c|c|c|c|c|c|c|c|c|c|}
\hline Name & Redshift & Type & $\begin{array}{c}S_{24} \\
(\mu \mathrm{Jy})\end{array}$ & CSC Name & $\begin{array}{c}\text { CSC 2-10 keV } \\
\text { Flux } \\
\left(\mathrm{erg} \mathrm{s}^{-1} \mathrm{~cm}^{-2}\right)\end{array}$ & $\begin{array}{c}\text { CSC HR } \\
\text { (h-s) }\end{array}$ & 2XMM Name & $\begin{array}{c}\text { 2XMM } 0.2-12 \mathrm{keV} \\
\text { Flux } \\
\left(\mathrm{erg} \mathrm{s}^{-1} \mathrm{~cm}^{-2}\right)\end{array}$ & 2XMM HR2 \\
\hline SW003312.39-431841.9 & 0.248 & 1 & 1030.0 & CXO J003312.3-431842 & $2.61 \mathrm{E}-14$ & 0.716161 & 2XMM J003312.3-431841 & $2.60 \mathrm{E}-14$ & 0.93102986 \\
\hline SW003316.92-431706.3 & & & 2200.0 & CXO J003316.9-431706 & $8.12 \mathrm{E}-15$ & 0.375333 & 2XMM J003317.0-431705 & 4.29E-14 & 0.917 \\
\hline SW003330.42-431554.3 & 0.437 & 3 & 1890.0 & CXO J003330.4-431554 & $3.77 \mathrm{E}-15$ & -0.325 & 2XMM J003330.4-431552 & $5.79 \mathrm{E}-15$ & 1.0 \\
\hline SW003333.75-432326.9 & & & 1490.0 & CXO J003333.7-432326 & $4.94 \mathrm{E}-15$ & 0.0643 & 2XMM J003333.7-432328 & $1.98 \mathrm{E}-14$ & 0.651 \\
\hline SW003336.26-431731.7 & & & 2190.0 & CXO J003336.2-431731 & $1.82 \mathrm{E}-14$ & 0.482 & 2ХMM J003336.1-431732 & $3.58 \mathrm{E}-14$ & 0.837 \\
\hline SW003346.28-431943.7 & 0.402 & 2 & 3240.0 & CXO J003346.2-431943 & $4.02 \mathrm{E}-14$ & 0.025 & 2ХMM J003346.2-431944 & $6.40 \mathrm{E}-14$ & 0.176 \\
\hline SW003357.32-433601.7 & 1.932 & 4 & 1060.0 & CXO J003357.2-433602 & $1.24 \mathrm{E}-14$ & -0.341 & & & \\
\hline
\end{tabular}

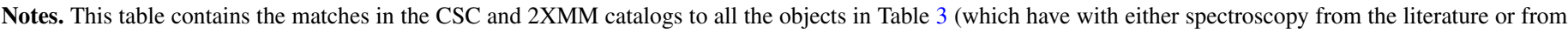
our own observations). Objects with featureless spectra are included (with the redshift and type columns left blank).

(This table is available in its entirety in a machine-readable form in the online journal. A portion is shown here for guidance regarding its form and content.)

broad-line and type 2 populations (although there is no obvious luminosity dependence in Figure 11). If this result is real, however, one interpretation can be obtained if we assume that the intrinsic mean ratio of mid-infrared to radio emission is the same for both populations, but that the type 2 s have some extinction toward the mid-infrared, even at $15 \mu \mathrm{m}$. This interpretation is broadly consistent with the work on radio-loud AGNs by Cleary et al. (2007) and Haas et al. (2008), where the infrared SEDs of radio galaxies and radio-loud quasars matched in radio luminosity differ by a factor $\approx 3$ at $12 \mu \mathrm{m}$ (i.e., very similar to our $\approx$ factor of two difference at $15 \mu \mathrm{m}$ ), despite having similar total far-infrared luminosities. This interpretation may also be supported by our tentative findings in Section 8 regarding the optical depth of the torus. However, this hypothesis must remain speculative pending better radio data and consideration of the full infrared SEDs and demographics of these objects.

\section{THE NATURE OF OBJECTS LACKING AGN SIGNATURES IN THE OPTICAL}

A significant fraction (22\%) of our objects with redshifts show no clear AGN signatures in their optical spectra and are classified as "non-AGNs." Where spectral features are seen, these objects have low-ionization, emission-line spectra. An additional 15\% of our candidates have featureless spectra or spectra with only a single, unidentifiable faint line (redshift quality $=4$ ). These candidates are excluded from our AGN sample, but may include
AGNs with both the narrow-line region (NLR) and the AGN continuum/broad-line region heavily obscured. In Figure 2, they are spread throughout the selection region, some close to the edges, suggesting that indeed they are interlopers, but some are directly along the AGN locus and satisfy the Donley et al. (2012) selection criteria. For a few of the objects, we have ancillary information indicating an AGN. Nine objects with "non-AGN" spectra are detected in the 2XMM or CSC catalogs, including SW021822.13-050614.1, which Severgnini et al. (2003) show has faint AGN emission lines in its nuclear spectrum once a host galaxy template is subtracted from a deep optical spectrum. Fourteen objects with optically featureless or single emission line spectra are detected in X-rays. Finally, one object is radio-intermediate, with $R_{I R}^{*}=0.13$ (SW160857.99+541818.4) and one object, XFLS171115.2+594906, has a flat radio spectrum, indicative of its radio emission arising from an AGN (a spectral index between $610 \mathrm{MHz}$ and $1.4 \mathrm{GHz}$ of $\alpha=-0.3$ ).

Based on comparing the X-ray detection rate of type 2 AGNs and objects lacking AGN signatures, we can place a rough lower bound on the number of AGNs missing in our optically classified sample. Forty-five optically classified type 2 AGNs are detected in X-rays, out of a total of 295 in the survey, a detection rate of $15 \%$. In contrasts, 23 objects have featureless spectra or otherwise no sign of an AGN in their optical spectra, out of a total of 233 such objects in the survey, a detection rate of $10 \%$, only a little lower than that of the type $2 \mathrm{~s}$. If we assume that the ratio 

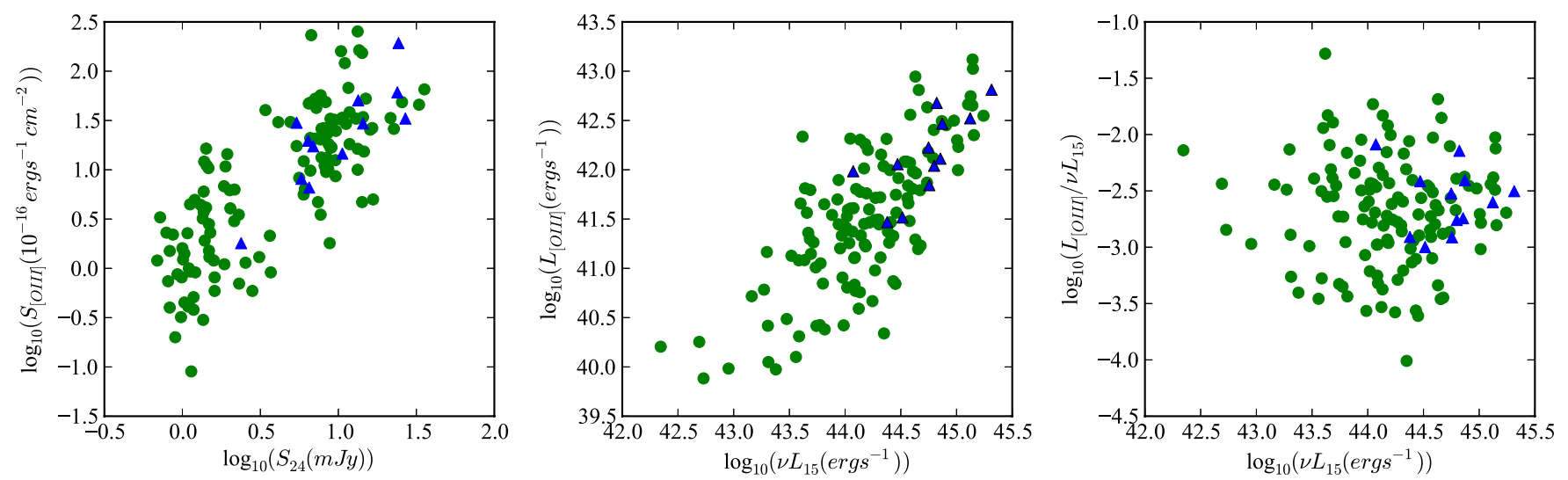

Figure 12. Left panel: $\log$ of [O III] 5007 flux, $S_{[\mathrm{O} I \mathrm{II}]}$ vs. $S_{24}$. Middle panel: $\log$ of [O III] $] 5007$ luminosity, $L_{[\mathrm{O} \text { III }]}$ vs. $15 \mu \mathrm{m}$ luminosity $\left(\nu L_{15}\right)$. Right panel: log of the ratio of [O III] to $15 \mu \mathrm{m}$ luminosity vs. $15 \mu \mathrm{m}$ luminosity. In all plots, type 2 AGNs are plotted as green dots and type 1 AGNs are plotted as blue triangles.

(A color version of this figure is available in the online journal.)

of X-ray detected to X-ray undetected AGNs is similar in the objects optically classified as "non-AGNs" as it is in the type 2 s, this result implies that at least $\approx 65 \%$ of the objects classified as "non-AGNs" on the basis of their optical spectra may in fact contain an AGN.

\section{THE EMISSION-LINE-MID-INFRARED LUMINOSITY CORRELATION}

Both the higher ionization emission lines and the mid-infrared emission are powered by UV emission from the AGN, so we expect these two quantities to correlate well. To test this idea, we measured the [O III]5007 flux in normal type 1 and type 2 AGN spectra at $z<0.75$, where this line is present in the optical spectra. As shown in Figure 12, a correlation exists between both fluxes and luminosities, with about a half dex scatter. The origin of this scatter is unclear. Although we see evidence of dust reddening in the type 2 composite (see Section 9), the fact that the type 1 and type 2 AGNs are seen to plot in the same region in Figure 12 and with similar scatter about the mean correlations suggests that reddening is not the primary cause of the scatter. Other possibilities include variation in ionization level of the NLR (unlikely given the similarity of the NLR spectra we see) or variation in the covering factor of dust in the nuclear region. This latter explanation seems the most likely, although it is surprising that there is no strong correlation of this ratio with luminosity. Such a correlation would naturally occur in the simplest version of the "receding torus" model (Lawrence 1991; Simpson 2006), where, in the case of a torus being optically thin in the mid-infrared, the mid-infrared luminosity should scale proportionally to the covering factor of hot dust, $\omega$, and the [O III] luminosity should scale proportionally to $(1-\omega)$. The large scatter means that our result is probably consistent with the relatively weak dependence of covering factor with AGN luminosity found by Roseboom et al. (2013), although our result may be inconsistent with the optically thin torus case of Lusso et al. (2013; indeed, Drouart et al. (2012) show that an optically thick torus is a good fit to radio galaxy SEDs when using the radio core-to-lobe flux ratio to constrain the inclination of the torus).

\section{COMPOSITE SPECTRA}

We constructed a composite spectrum for type 2 quasars and also the "non-AGN" class to search for evidence of weak AGN activity. Attempts to construct composites for the normal and reddened quasars did not result in useful spectra due to strong continua, which, particularly for the reddened quasars, varied considerably from object to object. The type 2 quasar composite was constructed using spectra from the bright samples (limiting fluxes $S_{24}>4 \mathrm{mJy}$ ) only at $z<0.8$ in order to exclude objects with Seyfert-like luminosities. At $z>0.8$, all samples contained objects with quasar-like luminosities, so all available spectra were combined. We used only spectra from our observations in Table 2 that were of good quality (signal-to-noise ratio of the brightest line $\gtrsim 30$ ) and which had good sky subtraction. Two of the fiber spectra, SW160929.35+542940.8 and SW160828.55+542546, had a low-order continuum subtraction performed before the addition since they had bad baselines due to poor sky subtraction.

The composite spectrum at wavelength $\lambda_{i}$ was constructed as

$$
F\left(\lambda_{i}\right)=\frac{1}{\sum_{j}\left(w_{j}\right)} \sum_{j} w_{j} a_{j} f_{j}\left(\lambda_{i}\right),
$$

where the sums are over all the spectra contributing at wavelength $\lambda_{i}$. The emission line flux scales approximately with the $24 \mu \mathrm{m}$ flux density $S_{24}$ (see Figure 12), so the scale factor $a_{j} \propto 1 / S_{24}^{j}$. The typical signal-to-noise, however, scales proportionally to $S_{24}$ (resulting in weights $w_{j} \propto S_{24}^{j}$ ).

The type 2 quasar spectrum (Figure 13) looks very similar to Seyfert-2 spectra, with strong high-ionization narrow lines, but it also includes low-ionization species such as [O I], consistent with the broad range of ionization expected from an AGN (although it lacks the coronal lines seen in the Rose et al. (2011) spectrum of SDSS J113111.05+162739.5). The composite host galaxy shows multiple Balmer absorption features, consistent with a dominant stellar population $\lesssim 10^{8} \mathrm{yr}$ old, as has been seen in the hosts of IR-luminous type 1 quasar hosts (Canalizo \& Stockton 2000).

The "non-AGN" spectrum (Figure 14) was constructed from an average of all the spectra for the objects lacking AGN features in their optical spectra; it is restricted to the optical as there are no examples at $z>1$ (at least not ones with detectable emission lines). The emission lines in both the individual spectra and the composite are more characteristic of starbursts than LINERs; as noted by L07, the number of candidate LINERs in these mid-infrared selected samples is very small and we have no definitive LINER candidates. We have carefully examined our composite spectrum for signs of high ionization lines that could indicate the presence of a weak or highly obscured AGN but 

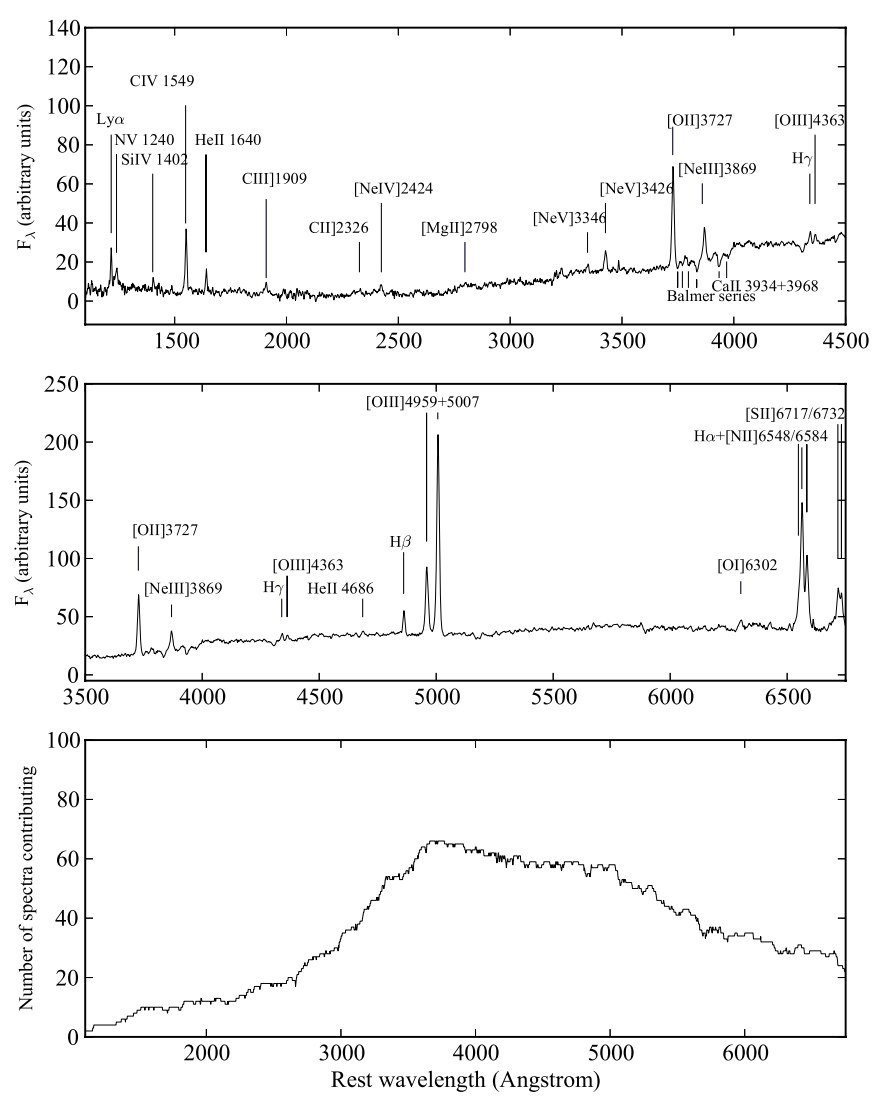

Figure 13. Type 2 quasar composite spectrum. The bottom panel shows the number of quasar spectra contributing at each wavelength.
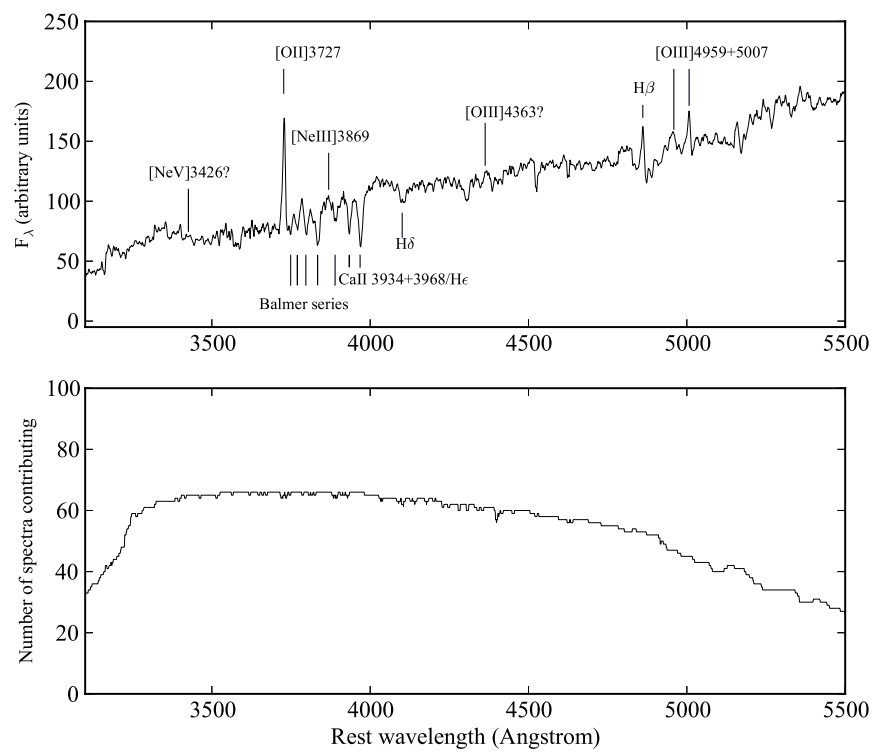

Figure 14. Composite spectrum of objects lacking AGN signatures in their individual spectra. The bottom panel shows the number of spectra contributing at each wavelength.

fail to find any conclusive evidence of these. Perhaps the best evidence from the composite that these objects are similar to the type $2 \mathrm{~s}$ is the very similar host galaxy spectra, including Balmer absorption lines of similar equivalent width at $\mathrm{H} 9$ and higher. These objects could thus be obscured AGNs with significant reddening toward the NLR, or simply lacking one. Nevertheless, we choose to exclude these objects from this AGN census until more unequivocal evidence for their AGNs is found.
Table 6

Line Ratios in Composite Spectra Relative to $\mathrm{H} \beta$

\begin{tabular}{|c|c|c|c|c|c|}
\hline Line & Wavelength & $\begin{array}{l}\text { Type } 2 \\
f / \mathrm{H} \beta\end{array}$ & Non-AGN & $\begin{array}{c}\text { Radio } \\
\text { Galaxy }^{\mathrm{a}} \\
f / \mathrm{H} \beta\end{array}$ & Case B \\
\hline $\operatorname{Ly} \alpha$ & 1216 & 0.75 & $\ldots$ & 31 & 31 \\
\hline $\mathrm{N} \mathrm{v}$ & 1240 & 0.93 & $\ldots$ & 1.5 & $\ldots$ \\
\hline Si IV & 1402 & 0.21 & $\ldots$ & 1.6 & $\ldots$ \\
\hline C IV & 1549 & 1.45 & $\ldots$ & 3.6 & $\ldots$ \\
\hline He II & 1640 & 0.40 & $\ldots$ & 3.2 & $\ldots$ \\
\hline $\left.\mathrm{C}_{\mathrm{III}}\right]$ & 1909 & 0.34 & $\ldots$ & 1.8 & $\ldots$ \\
\hline $\left.\mathrm{C}_{\mathrm{II}}\right]$ & 2326 & 0.16 & $\ldots$ & 0.92 & $\ldots$ \\
\hline $\mathrm{Ne}$ IV] & 2424 & 0.40 & $\ldots$ & 0.90 & $\ldots$ \\
\hline Mg II & 2798 & 0.75 & $\ldots$ & 0.78 & $\ldots$ \\
\hline $\mathrm{Ne} v]$ & 3346 & 0.16 & $\ldots$ & 0.20 & $\ldots$ \\
\hline $\mathrm{Ne} v]$ & 3426 & 0.53 & $<0.1$ & 0.69 & $\ldots$ \\
\hline O II] & 3727 & 2.78 & 2.5 & 3.64 & $\ldots$ \\
\hline $\mathrm{Ne}$ III] & 3869 & 0.93 & 0.33 & 0.82 & $\ldots$ \\
\hline $\mathrm{H} \gamma$ & 4340 & 0.19 & $\ldots$ & 0.24 & 0.47 \\
\hline O III] & 4363 & 0.18 & $<0.25$ & 0.08 & $\ldots$ \\
\hline He II & 4686 & 0.18 & $\ldots$ & 0.20 & $\ldots$ \\
\hline $\mathrm{H} \beta$ & 4861 & 1.0 & 1.0 & 1.0 & 1.0 \\
\hline O III] & 4959 & 3.9 & 0.38 & 3.1 & $\ldots$ \\
\hline O III] & 5007 & 10.8 & 0.99 & 8.7 & - \\
\hline O I] & 6302 & 0.41 & $\ldots$ & $\ldots$ & $\ldots$ \\
\hline N II] & 6548 & 1.4 & $\ldots$ & $\ldots$ & $\ldots$ \\
\hline $\mathrm{H} \alpha$ & 6563 & 7.0 & $\ldots$ & $\ldots$ & 2.9 \\
\hline $\mathrm{N}$ II] & 6584 & 4.2 & $\ldots$ & $\ldots$ & $\ldots$ \\
\hline S II] & 6717 & 2.3 & $\ldots$ & $\ldots$ & $\ldots$ \\
\hline $\left.\mathrm{S}_{\mathrm{II}}\right]$ & 6732 & 2.0 & $\ldots$ & $\ldots$ & $\ldots$ \\
\hline
\end{tabular}

Note. ${ }^{a}$ From McCarthy (1993).

Table 6 compares the emission line fluxes from our type 2 and non-AGN composites with the radio galaxy template from McCarthy (1993) and a Case B recombination model (for the hydrogen lines). Our type 2 composite spectrum shows evidence for reddening, in terms of significant Balmer decrements between $\mathrm{H} \alpha, \mathrm{H} \beta$, and $\mathrm{H} \gamma$, and a weak $\mathrm{Ly} \alpha$ line. There are also differences between the type 2 composite and that of the radio galaxies; in particular, the low-ionization UV lines (e.g., $\mathrm{C}$ III] and $\mathrm{C}$ II] are weak compared with $\mathrm{C}_{\text {IV }}$ in our composite when compared to radio galaxies) and the Ly $\alpha$ line is much weaker in the type 2 spectrum than in the radio galaxy composite. We speculate that this fact may be due to the effect of jet-induced shocks in the radio galaxies (a result consistent with the morphologies of extended emission line nebulae of type 2 quasars studied by Liu et al. 2013), but defer a detailed analysis to a future paper.

\section{DISCUSSION}

Our survey of 786 objects with (attempted) spectra contains 672 extragalactic objects with redshifts ( 24 objects are stars included in error due to saturated flux densities in the IRAC bands and 90 objects had featureless spectra). Of these, we have classified 136 as type 1 (normal quasars/Seyfert 1 s), 96 as type 1 objects showing significant signs of reddening, 294 as type 2 quasars/Seyfert 2s, leaving 145 objects with redshifts but showing no sign of AGN activity in their optical spectra. Our nested survey strategy has enabled us to span a wide range in luminosity (>a factor of 10) at a given redshift. We classify $340(50 \%)$ of the survey AGNs as quasars based on their mid-infrared luminosities indicating that their bolometric accretion luminosities are $\gtrsim 10^{12} L_{\odot}$. The difference in extinction in the mid-infrared 
between type 1 and type 2 objects is probably at most a factor of $\approx 3$ (see the discussion in Section 6). Thus, we are able to select very comparable objects with both normal type 1 and obscured quasar natures in the rest-frame optical.

We defined a "statistical survey" with $90 \%$ complete redshift information in order to allow for the inevitable bias toward type 1 objects in our spectroscopic survey. Members of this survey were identified by ordering each sample contributing to the survey in $24 \mu \mathrm{m}$ flux density from high to low and including objects in the statistical survey by working down the list until the spectroscopic completeness fell below 90\% (if at all). This sample consists of 662 objects, with 122 normal type 1s, 86 reddened type 1s, 271 type 2 s, and 118 "nonAGNs." Twenty-three objects were stars and 42 objects lacked redshifts. Obscured AGNs thus dominate the population over the redshift and luminosity range probed by this survey. We will use this survey to discuss the demographics and evolution of these objects in a future paper (S. E. Ridgway et al. 2013, in preparation).

The composite spectrum of the type 2 quasars shows high ionization narrow lines, but is different in the ultraviolet to radio galaxies, with weaker Ly $\alpha$ and other low-ionization species. The [O III]5007 line emission of our objects correlates well with their $24 \mu \mathrm{m}$ luminosity, and both type 1 and type 2 objects lie on the same correlation, showing that mid-infrared selection is indeed capable of selecting type 1 and type 2 quasars with similar intrinsic properties, with no apparent strong dependence of dust covering factor on AGN luminosity.

A significant fraction (259/785, or 33\%) of our AGN candidates showed no evidence for an AGN in terms of emission line diagnostics, either because the emission lines were detected but did not show AGN-like line ratios or because the spectra had at most only a single weak emission line. Twenty-five of these candidates have some other evidence for an AGN (X-ray emission or AGN-related radio emission) and an X-ray detection rate not much lower than the type 2 quasars. Overall, the composite spectrum shows that the stellar population of their host galaxies seems similar to that of the type 2 quasars. Most of these objects may therefore be AGNs whose NLRs are obscured, but as we cannot prove this statement (except in $\approx 10 \%$ of cases where we have other diagnostics) and these objects are excluded from our AGN statistics.

This study reinforces the need for multiple AGN diagnostics to be employed to complete a census of AGNs, even for surveys of luminous AGNs and quasars such as this one. Nevertheless, our sample will provide important insights into the luminosity dependence and cosmic evolution of obscuration. The publication of the Wide-field Infrared Survey Explorer (Wright et al. 2010) all-sky survey will also allow us to continue this survey into even brighter regimes, which will be particularly useful in understanding the luminosity dependence of obscuration at $z<1$.

N.S. is the recipient of an Australian Research Council Future Fellowship. M.L., A.P., and T.U. were visiting astronomers at the Infrared Telescope Facility, which is operated by the University of Hawaii under cooperative agreement no. NNX-08AE38A with the National Aeronautics and Space Administration (NASA), Science Mission Directorate, Planetary Astronomy Program. The Gemini Observatory is operated by the Association of Universities for Research in Astronomy, Inc, under a cooperative agreement with the National Science Foundation on behalf of the Gemini Partnership. Partly based on observations obtained at the Southern Astrophysical Research (SOAR) telescope, which is a joint project of the Ministério da Ciência, Tecnologia, e Inovação (MCTI) da República Federativa do Brasil, the U.S. National Optical Astronomy Observatory (NOAO), the University of North Carolina at Chapel Hill (UNC), and Michigan State University (MSU). We thank the Telescope System Instrumentation Program (TSIP), administered by NOAO, for the opportunity to observe on the Multiple Mirror Telescope (MMT). This research has made use of the NASA/IPAC Extragalactic Database (NED), which is operated by the Jet Propulsion Laboratory, California Institute of Technology, under contract with the National Aeronautics and Space Administration. This research has made use of data obtained from the Chandra Source Catalog, provided by the Chandra X-Ray Center (CXC) as part of the Chandra Science Archive. The NRAO is a facility of the National Science Foundation operated under cooperative agreement by Associated Universities, Inc. This paper is dedicated to the late Steve Rawlings, whose work on radio galaxy samples directly inspired the approach taken to this project.

\section{APPENDIX \\ NOTES ON INDIVIDUAL OBJECTS}

\section{A.1. SW021749.00-052306.9}

This object had a redshift of 0.987 assigned by L07 on the basis of the detection of a single emission line, presumed to be [O II]3727. Lanzuisi et al. (2009) obtained an improved spectrum of this object, showing that the redshift is in fact 0.914 (consistent with the line we identified by L07 being [Ne III]3869 rather than [O II]). Lanzuisi et al. also detect strong [O III]5007 emission beyond the wavelength range of our original spectrum, confirming it as a type 2 AGN.

\section{A.2. SW021928.77-045433.7}

There is a clear detection of an emission line in the $H$-band spectrum from Gemini, but other features are less certain. The $[\mathrm{NeV}] 3426$ line is in a region of poor atmospheric transmission, so it may be a noise spike. The optical spectrum, however, shows marginal evidence of a spectral break around the wavelength of Ly $\alpha$ if the redshift is 3.23 , and a marginally significant feature corresponding CIV1549 if the line in the $H$-band is [O II] 3727. We therefore assign this object a redshift of 3.23 with a redshift quality of 2 .

\section{A.3. SW021822.13-050614.1}

This low redshift $(z=0.044)$ object is an X-ray source. Optically, it is classified as a non-AGN, but, as discussed by Severgnini et al. (2003), its true nature is an AGN.

\section{A.4. SW021947.53-051008.5}

This quasar shows a blueshifted He I line in absorption (Figure 3). Leighly et al. (2011) discuss a broad absorption line quasar with He I absorption that may be similar to this object.

\section{A.5. SW022003.58-045145.6}

This object is a highly dust-reddened quasar at $z=1.33$ (Figure 3). The Hale and Gemini near-infrared spectra show only a single broad emission line at $1.53 \mu \mathrm{m}$, however, a Keck/ LRIS spectrum shows several UV emission lines at a redshift of $z=1.33$. 


\section{A.6. SW104159.83+585856.4}

This otherwise unremarkable $z=0.342$ type 2 quasar has an anomalous emission line at $4331 \AA$ that may correspond to an emission line from a higher redshift galaxy lensed by the quasar.

\section{A.7. SW105201.92+574051.5}

This object was independently identified by Coppin et al. (2010) as a high redshift, submillimeter bright obscured quasar (AzLOCK.01 in that paper). The Spitzer Infrared Spectrograph spectrum obtained by Coppin et al. shows both strong PAH features and a strong continuum at $z \approx 2.5$. Our Gemini/NIRI spectrum (Figure 4) shows a narrow $\mathrm{H} \alpha$ emission line at $z=2.467$.

\section{A.8. XFLS $171053.51+594433.1$}

This object, the second-highest redshift quasar in the sample, was first identified by Marleau et al. (2007) in a survey of radio emitters in the XFLS. The quasar has a flux density of $2.4 \mathrm{mJy}$ at $1.4 \mathrm{GHz}$, so is radio-intermediate in nature. The optical spectrum in Figure 3 of Marleau et al. shows only narrow $\operatorname{Ly} \alpha$ and NV1240, but our Hale spectrum with Triplespec (Figure 6) clearly shows a broad $\mathrm{H} \beta$ emission line, hence its classification as a reddened type 1 quasar.

\section{A.9. XFLS171419.9+602724}

This object, a $z=2.99$ type 2 quasar, is discussed in detail in Lacy et al. (2011).

\section{A.10. XFLS171754.6+600913}

At $z=4.27$, this object is the highest redshift object in our sample and the most luminous. It is a dust-reddened type 1 quasar with a mini-LoBAL system (Figure 6) with an overall luminosity of $\approx 10^{14} L_{\odot}$. Its Chandra X-ray detection falls well below the X-ray- $15 \mu \mathrm{m}$ luminosity correlation, presumably due to absorption.

\section{REFERENCES}

Abazajian, K. N., Adelman-McCarthy, J. K., Agüeros, M. A., et al. 2009, ApJS, 182,543

Appleton, P. N., Fadda, D. T., Marleau, F. R., et al. 2004, ApJS, 154, 147

Berta, S., Rubele, S., Franceschini, A., et al. 2006, A\&A, 451, 881

Bertin, E., \& Arnouts, S. 1996, A\&AS, 117, 393

Brightman, M., \& Nandra, K. 2011, MNRAS, 414, 3084

Brown, M. J. I., Brand, K., Dey, A., et al. 2006, ApJ, 638, 88

Burgers, A. M., \& Hunstead, R. W. 2006, AJ, 131, 114

Canalizo, G., \& Stockton, A. 2000, AJ, 120, 1750

Ciliegi, P., McMahon, R. G., Miley, G.K., et al. 1999, MNRAS, 302, 222

Cleary, K., Lawrence, C. R., Marshall, J. A., Hao, L., \& Meier, D. 2007, ApJ, 660, 117

Colless, M. M., Dalton, G., Maddox, S., et al. 2001, MNRAS, 328, 1039

Condon, J. J., Cotton, W. D., Yin, Q. F., et al. 2003, AJ, 125, 2411

Coppin, K., Pope, A., Menéndez-Delmestre, K., Alexander, D. M., \& Dunlop, J. 2010, ApJ, 713, 503

Cutri, R. M., Nelson, B. O., Francis, P. J., \& Smith, P. S. 2002, in IAU Colloq. 184, ASP Conf. Ser. 284, AGN Surveys, ed. R. F. Green, E. Ye. Khachikian, \& D. B. Sanders (San Francisco, CA: ASP), 127

Cutri, R. M., Strutskie, M. F., van Dyk, S., et al. 2003, The IRSA 2MASS All-Sky Point Source Catalog, NASA/IPAC Infrared Science Archive, http://irsa.ipac.caltech.edu/applications/Gator/

Dennefeld, M., Lagache, G., Mei, S., et al. 2005, A\&A, 440, 5

de Vaucouleurs, G., de Vaucouleurs, A., Corwin, H. G., et al. 1991, Third Reference Catalog of Bright Galaxies (New York: Springer)

di Serego-Alighieri, S., Danziger, I. J., Morganti, R., \& Tadhunter, C. N. 1994, MNRAS, 269, 998

Donley, J. L., Koekemoer, A. M., Brusa, M., et al. 2012, ApJ, 748, 142
Donley, J. L., Rieke, G. H., Rigby, J. R., \& Pérez-González, P. G. 2005, ApJ, 634,169

Drouart, G., de Breuck, C., Vernet, J., et al. 2012, A\&A, 548, 45

Evans, I. N., Primini, F. A., Glotfelty, K. J., et al. 2010, ApJS, 189, 37

Fadda, D., Marleau, F. R., Storrie-Lombardi, L. J., et al. 2006, AJ, 131, 2859

Glikman, E., Gregg, M. D., Lacy, M., Helfand, D. J., Becker, R. H., \& White, R. L. 2004, ApJ, 607, 60

Glikman, E., Helfand, D. J., White, R. L., et al. 2007, ApJ, 667, 673

Gonzáles-Solares, E. A., Irwin, M., McMahon, R. G., et al. 2011, MNRAS, 416, 927

Gregg, M. D., Lacy, M., White, R. L., et al. 2002, ApJ, 564, 133

Gwyn, S. D. J. 2012, AJ, 143, 38

Haas, M., Willner, S. P., Heymann, F., et al. 2008, ApJ, 688, 122

Herter, T. L., Henderson, C. P., Wilson, J. C., et al. 2008, Proc. SPIE, 7014, 30

Ibar, E., Ivison, R. J., Biggs, A. D., et al. 2009, MNRAS, 397, 281

Jarvis, M., Bonfield, D. G., Bruce, V. A., et al. 2013, MNRAS, 428, 1281

Johansson, P. H., Väisänen, P., \& Vaccari, M. 2004, A\&A, 427, 795

Jones, D. H., Read, M. A., Saunders, W., et al. 2009, MNRAS, 399, 683

Kimball, A. E., Kellerman, K. I., Condon, J. J., et al. 2011, ApJ, 739, L29

Kocevski, D. D., Lubin, L. M., Lemaux, B. C., et al. 2012, ApJ, 744, 148

Lacy, M., Gregg, M. D., Becker, R. H., et al. 2002, AJ, 123, 2925

Lacy, M., Petric, A. O., Martíez-Sansigre, A., et al. 2011, AJ, 142, 196

Lacy, M., Petric, A. O., Sajina, A., et al. 2007a, AJ, 133, 186 (L07)

Lacy, M., Sajina, A., Petric, A. O., et al. 2007b, ApJ, 669, L61

Lacy, M., Sajina, A., Petric, A. O., et al. 2012, in IAU Symp. 284, The Spectral Energy Distribution of Galaxies, ed. R. J. Tuffs \& C. C. Popescu (Cambridge: Cambridge Univ. Press), 224

Lacy, M., Storrie-Lombardi, L. J., Sajina, A., et al. 2004, ApJS, 154, 166

Lacy, M., Wilson, G., Masci, F., et al. 2005, ApJS, 161, 41

La Franca, F., Gruppioni, C., Matute, I., et al. 2004, AJ, 127, 3075

Lanzuisi, G., Piconcelli, E., Fiore, F., et al. 2009, A\&A, 498, 67

Lawrence, A. 1991, MNRAS, 248, 91

Lawrence, A., Warren, S. J., Almaini, O., et al. 2007, MNRAS, 379, 1599

Leighly, K. M, Dietrich, M., \& Barber, S. 2011, ApJ, 728, L94

Lonsdale, C. J., Smith, H. E., Rowan-Robinson, M., et al. 2003, PASP, 115, 897

Liu, G., Zakamska, N. L., Greene, J. E., Nesvadba, N. P. H., \& Liu, X. 2013, MNRAS, 430, 2327

Lusso, E., Hennawi, J. F., Comastri, A., et al. 2013, ApJ, in press (arXiv:1309.0814)

Maddox, S. J., Sutherland, W. J., Efstathiou, G., \& Loveday, J. 1990, MNRAS, 243, 692

Magliocchetti, M., Maddox, S. J., Wall, J. V., Benn, C. R., \& Cotter, G. 2000, MNRAS, 318, 1047

Manners, J. C., Serjeant, S., Bottinelli, S., et al. 2004, MNRAS, 355, 97

Mao, M., Sharp, R., Norris, R. P., et al. 2012, MNRAS, 426, 3334

Marleau, F., Fadda, D., Appleton, P. N., et al. 2007, ApJ, 663, 218

Martínez-Sansigre, A., Rawlings, S., Lacy, M., et al. 2005, Natur, 436, 666

Martínez-Sansigre, A., Rawlings, S., Lacy, M., et al. 2006, MNRAS, 370, 1479

Martínez-Sansigre, A., \& Taylor, A. M. 2009, ApJ, 692, 964

Mauduit, J.-C., Lacy, M., Farrah, D., et al. 2012, PASP, 124, 1135

McCarthy, P. J. 1993, ARA\&A, 31, 639

Middelberg, E., Norris, R. P., Cornwell, T. J., et al. 2008, AJ, 135, 1276

Morel, T., Efstathiou, A., Serjeant, S., et al. 2001, MNRAS, 327, 1187

Norman, C., Hasinger, G., Giacoconi, R., et al. 2002, ApJ, 571, 218

Norris, R. P., Afonso, J., Appleton, P. N., et al. 2006, AJ, 132, 2409

Ogle, P., Whysong, D., \& Antonucci, R. 2006, ApJ, 647, 161

Oyabu, S., Ynun, M. S., Murayama, T., et al. 2005, AJ, 130, 2019

Papovich, C., Cool, R., Eisenstein, D., et al. 2006, AJ, 132, 231 (P06)

Park, S. Q., Barmby, P., Fazio, G. G., et al. 2008, ApJ, 678, 744

Polletta, M., Omont, A., Berta, S., et al. 2008, A\&A, 492, 81

Rayner, J. T., Toomey, D. W., Onaka, P. M., et al. 2003, PASP, 115, 362

Reyes, R., Zakamska, N. L., Strauss, M. A., et al. 2008, AJ, 126, 2373

Richards, G. T., Hall, P. B., Vanden Berk, D. E., et al. 2003, AJ, 166, 1131

Richards, G. T., Lacy, M., Storrie-Lombardi, L. J., et al. 2006, ApJS, 166, 470

Robert, C., Leitherer, C., \& Heckman, T. 1993, ApJ, 418, 749

Rose, M., Tadhunter, C. N., Holt, J., Ramos Almeida, C., \& Littlefair, S. P. 2011, MNRAS, 414, 3360

Roseboom, I. G., Lawrence, A., Elvis, M., et al. 2013, MNRAS, 429, 1494

Rowan-Robinson, R., Babbedge, T., Surace, J., et al. 2005, AJ, 129, 1183

Sanders, D. B., Salvato, M., Aussel, H., et al. 2007, ApJS, 172, 86

Sajina, A., Lacy, M., \& Scott, D. 2005, ApJ, 621, 256

Schechtman, S. A., Landy, S. D., Oemler, A., et al. 1996, ApJ, 470, 172

Schmidt, M., Hasinger, G., Gunn, J., et al. 1998, A\&A, 329, 495

Serjeant, S., Efstathiou, A., Oliver, S., et al. 2001, MNRAS, 322, 262

Severgnini, P., et al. 2003, A\&A, 406, 483

Shapley, A. E., Steidel, C. C., Pettini, M., \& Adelberger, K. L. 2003, ApJ, 588, 65 
Sharp, R. G., Sabbey, C. N., Vivas, A. K., Oemler, A., et al. 2002, MNRAS, $337,1153(\mathrm{Sh} 02)$

Simpson, C., Martínez-Sansigre, A., Rawlings, S., et al. 2006, MNRAS, 372 , 741 (S06)

Simpson, C., Rawlings, S., Ivison, R., et al. 2012, MNRAS, 421, 3060 (S12) Soltan, A. 1982, MNRAS, 200, 115

Sopp, H. M., \& Alexander, P. 1991, MNRAS, 251, 14

Spinrad, H., Djorgovski, S., Marr, J., \& Aguilar, L. 1985, PASP, 97, 932

Stern, D., Eisenhardt, P., Gorjian, V., et al. 2005, ApJ, 631, 163

Strutskie, M. F., Cutri, R. M., Stiening, R., et al. 2006, AJ, 131, 1163

Sturm, E., Rupke, D., Contursi, A., et al. 2006, ApJ, 653, L13

Teng, S. H., Wilson, A. S., Veilleux, S., et al. 2005, ApJ, 633, 664

Treister, E., Schawinski, K., Urry, C. M., \& Simmons, B. D. 2012, ApJ, 758, L39

Trump, J. R., Impey, C. D., McCarthy, P. J., et al. 2007, ApJS, 172, 383
Urrutia, T., Becker, R. H., White, R. L., et al. 2009, ApJ, 698, 1095

Urrutia, T., Lacy, M., \& Becker, R. H. 2008, ApJ, 674, 80

Urrutia, T., Lacy, M., Spoon, H., et al. 2012, ApJ, 757, 125

Watson, M. G., Schröder, A. C., Fyfe, D., et al. 2009, A\&A, 493, 339

Webster, R. L., Francis, P. J., Peterson, B. A., Drinkwater, M. L., \& Masci, F. J. 1998, Natur, 375, 469

White, R. L., Becker, R. H., Gregg, M. D., et al. 2000, ApJS, 126, 133

White, R. L., Helfand, D. J., Becker, R. H., Glikman, E., \& de Vries, W. 2007, ApJ, 654, 99

Whiting, M. T., Webster, R. L., \& Francis, P. J. 2001, MNRAS, 323, 718

Wisotzki, L., Cristlieb, N., Bade, N., et al. 2000, A\&A, 358, 77

Wright, E., Eisenhardt, P. R. M., \& Mainzer, A. K. 2010, AJ, 140, 1868

Yan, L., Sajina, A., Fadda, D., et al. 2007, ApJ, 658, 778

Zakamska, N. L., Strauss, M. A., Krolik, J. H., et al. 2003, AJ, 126 2125 